\title{
Critical Behavior of a Charged Bose Gas
}

\author{
R. F. Bishop* \\ Department of Theoretical Physics, The Schuster Laboratory. \\ Manchester University, Manchester, England
}

(Received November 26, 1973)

A fully self-consistent Hartree-Fock theory, using the Coulomb interaction screened by the polarization insertions calculated in the self-consistent randomphase approximation, is applied to the d-dimensional, dense, charged Bose gas at temperatures close to the transition temperature $T_{\mathrm{c}}$. The quasiparticle energy spectrum is calculated and shown to behave at $\mathrm{T}_{\mathrm{c}}$ like $\mathrm{\varepsilon}(\mathrm{k})=\mathrm{Ak}^{\sigma}$ for small $\mathrm{k}$, and $\sigma$ is calculated as a function of the dimensionality $\mathrm{d}$. The change in transition temperature from that of an ideal gas at the same density, and of the chemical potential are shown to be given by $\left(\mathrm{T}_{\mathrm{c}}-\mathrm{T}_{\mathrm{c} 0}\right) / \mathrm{T}_{\mathrm{c} 0} \approx \mathrm{Xr}_{\mathrm{s}}^{(\mathrm{d}-2) / 3}$ and $\mu_{\mathrm{c}} \approx \mathrm{Yr}_{\mathrm{s}}^{2 / 3}$, where $\mathrm{r}_{\mathrm{s}}$ is the ratio of the interparticle spacing to the Bohr radius. Approximate expressions are given for the coefficients $\mathrm{X}$ and $\mathrm{Y}$. The critical exponents are calculated, and the system is shown to obey exact scaling.

\section{INTRODUCTION}

The charged Bose gas was first investigated theoretically (at zero temperature) by Foldy, ${ }^{1}$ who showed that the Coulomb interaction becomes relatively easy to handle in the high-density limit, just as in the case of the charged Fermi gas. ${ }^{2}$ In this limit a small parameter $r_{s}$ appears in the problem, equal to the ratio of the interparticle spacing to the Bohr radius. Using a Bogulyubov transformation, ${ }^{3}$ Foldy evaluated the leading order expression for the ground-state energy per particle at zero temperature in this limit, and found it to be proportional to $r_{s}^{-3 / 4}$. Other authors ${ }^{4-9}$ have extended the zero-temperature formalism to verify these results and to obtain the next few terms in the series expansion in (fractional) powers of $r_{s}$. Similar methods have also been employed to study the charged Bose gas in the high-temperature limit. ${ }^{2,10}$

The very interesting region of intermediate temperatures has only begun to be studied recently. ${ }^{11-13}$ One knows that an ideal Bose gas undergoes

*This work was completed while the author held an SRC Postdoctoral Research Fellowship. 
a phase transition at a critical temperature $T_{c 0}$ below which the system contains a condensate of particles macroscopically occupying a single state, and it is of considerable theoretical interest to investigate the effects of interparticle interactions on this transition. Choosing the interaction to be a pure Coulomb repulsion provides the theorist with a nontrivial, but analytically simple example of an interacting boson assembly. The model serves as a classic example of a system with long-range interaction, and one may hope that some of its properties may be shared by systems interacting via more complicated interactions containing a long-range part. In particular, we shall explicitly demonstrate in our calculation those features of the system encountered in the transition region that arise from the overlap of the lowmomentum singularity in the interaction with the corresponding singularity in the boson distribution function at small values of the momentum.

The charged Bose gas has also some interest as a model of several real physical systems. For example, many years ago Ginzburg ${ }^{14}$ and Schafroth ${ }^{15}$ realized that an ideal charged Bose gas (i.e., where the Coulomb interaction is ignored) displays a Meissner effect at temperatures below $T_{c 0}$, and could thus serve as a model for superconductors. It has since been shown that the inclusion of the Coulomb interaction does not destroy the Meissner effect, ${ }^{16}$ although the electrodynamics of the system becomes essentially local in the high-density limit ${ }^{17}$ as compared with the nonlocal electrodynamics of a BCS superconductor. ${ }^{18}$ More recently it has also been suggested that the charged Bose gas below its condensation temperature might be important in the study of some systems of astrophysical interest, ${ }^{19,20}$ such as, for example, white dwarf stars, which are presumed to consist largely of ionized helium.

Also, in recent years an enormous amount of work has been expended on the general theory of phase transitions, and in particular on the investigation of the critical indices ${ }^{21,22}$ that describe the singular behavior of the various thermodynamic functions in the vicinity of the transition. These investigations have now received added impetus from the very recent work of Wilson ${ }^{23}$ and its extension and application by other authors. In this context the charged Bose gas provides a simple theoretical model of a system with long-range repulsive interactions that exhibits critical behavior, and it can be used as a testing ground for various theoretical approaches. Thus, for example, the methods of Wilson ${ }^{23}$ have been applied to this system by Ma. ${ }^{12}$

In the present work the charged Bose gas is examined in the highdensity limit at temperatures equal to and above the critical point, and the whole formalism is carried through with the dimensionality $d$ of the system as an arbitrary (and continuous) variable. A fully self-consistent set of approximations is made, and these presumably become exact as the density becomes infinite. The formalism employed is that of finite-temperature 
("imaginary-time") many-body theory, and attention is focused on the oneand two-body Green's functions, which are used to derive the thermodynamic functions. Within the set of approximations made, the critical behavior of the system is identified explicitly, by calculating all of the critical exponents. It is demonstrated that the system obeys exact scaling, and all of the usual scaling relations are shown to be satisfied.

In the next section the system and its parameters are defined, and the various thermodynamic functions are set up in a manner convenient to the problem. The many-body theory formalism used is described in Section 3, and the necessary Green's functions are defined and related to the thermodynamics of the system. The critical exponents are defined in terms of the limiting behavior of these Green's functions. The self-consistent approximation that allows the problem to become tractable is made in terms of the same Green's functions, and is described in Section 4. It comprises, basically, the random phase approximation for the Coulomb potential, together with the Hartree-Fock approximation for the resulting screened interaction. Both of these approximations are made fully self-consistent, which is vital to any treatment of the critical region. It is shown explicitly that the resulting set of approximations is both self-consistent and consistent with the various exact relations for the thermodynamic functions of the system obtained from the Green's functions.

The resulting set of coupled integral equations is examined in the highdensity limit and at the critical temperature $T_{c}$, in Section 5. It is shown that the quasiparticle spectrum takes a particular form, which can be obtained from the solution to a single integral equation. The form of the spectrum at low momentum is shown to be $\varepsilon(k) \sim k^{\sigma}$, where $\sigma<2$; and the exponent $\sigma$ is solved for explicitly as a function of the dimensionality $d$.

The change in transition temperature $T_{c}-T_{\mathfrak{c} 0}$ from the ideal Bose gas is investigated in Section 6, and it is shown that at high density $T_{c}-T_{c 0}=$ $A r_{s}^{(d-2) / 3}$ to leading order in the parameter $r_{s}$. There is seen to be no phase transition for $d \leq 2$, just as for the ideal gas. The chemical potential $\mu_{\mathrm{c}}$ at the critical point is shown to behave as a function of density like $\mu_{c}=B r_{s}^{2 / 3}$ for all $d$, at high density. The numerical constants $A$ and $B$ are related to the form of the quasiparticle spectrum for all values of the momentum, and approximate expressions are given from the knowledge of the low-momentum form only, found in the previous section.

The treatment of the self-consistent set of equations is extended in Section 7 to temperatures $T>T_{c}$, where it is shown explicitly that the critical behavior of the system near the transition region can be found exactly from a knowledge of the quasiparticle spectrum at low momentum values only. Thus, all of the critical exponents are identified as explicit functions of $d$ and the exponent $\sigma$ found in Section 5. It is demonstrated that the system in 
this set of approximations obeys exact scaling, and all of the usual scaling relations are verified.

A brief summary of the results is given in Section 8, and some integrals needed in the work are evaluated in the appendix.

\section{THERMODYNAMICS AND NOTATION}

The system under consideration is taken to be a large number $N$ of identical spinless charged bosons, of charge $e$ and mass $m$, together with a uniform background of opposite charge to ensure that the whole system is electrically neutral. The entire system is constrained to occupy a volume $\Omega$ in $d$ dimensions. It has become common in recent years in the context of phase transitions to treat the number of spatial dimensions $d$ which the system is constrained to occupy as a continuous variable. For example, Wilson ${ }^{23}$ has developed an analysis of phase transitions in which the quantity $\varepsilon=4-d$ is treated as a perturbation parameter. For generality, and to compare with such work, we shall also treat $d$ as a continuous parameter. In such case an infinitesimal volume element in $d$ dimensions is given by

$$
(2 \pi)^{-d} d^{d} x=(2 \pi)^{-1} K_{d-1} x^{d-1} d x \sin ^{d-2} \theta d \theta ; \quad 0 \leq \theta \leq \pi
$$

or

$$
(2 \pi)^{-d} d^{d} x=K_{d} x^{d-1} d x
$$

where

$$
K_{d}=2^{1-d} \pi^{-d / 2} / \Gamma\left(\frac{1}{2} d\right)
$$

where in Eq. (1b) the integration over the angular variable $\theta$ has been performed, and it is necessary that $d>1$ for the integral to exist.

We define a parameter $r_{s}=r_{0} / a_{0}$, where $r_{0}$ is the average interparticle spacing, and $a_{0}=\hbar^{2} / m e^{2}=2 / e^{2}$ is the Bohr radius. ${ }^{*}$ The high-density criterion is then quantified by requiring $r_{s} \ll 1$. The particle density $n$ is given by

$$
n^{-1}=r_{0}^{d} V_{d}(1)
$$

where $V_{d}(1)$ is the volume of a unit sphere in $d$ dimensions, which from Eq. (1) is given by

$$
V_{d}(1)=(2 \pi)^{d} K_{d} / d
$$

The parameter $r_{s}$ is thus given by

$$
e^{2}=2 r_{s}\left[n V_{d}(1)\right]^{1 / d}
$$

*Henceforth we employ a system of units such that $h=2 m=\Omega=1$. 
The interaction potential is taken to be

$$
V(r)=e^{2} r^{-1}
$$

and its $d$-dimensional Fourier transform is derived in the appendix. From Eq. (A6) we find

$$
V(k)=\lim _{\mu \rightarrow 0} e^{2} \Gamma\left(\frac{1}{2} d-\frac{1}{2}\right)\left(\frac{4 \pi}{k^{2}+\mu^{2}}\right)^{d / 2-1 / 2}
$$

where $\mu$ is a spatial cutoff introduced as a standard artifice to enable the transform to be defined. As usual, the uniform background charge of opposite sign to that of the bosons exactly cancels the term in $V(k)$ with $k=0$, and we get

$$
V(k)= \begin{cases}Z_{d} e^{2} k^{1-d}, & k \neq 0 \\ 0, & k=0\end{cases}
$$

where

$$
Z_{d}=(4 \pi)^{d / 2-1 / 2} \Gamma\left(\frac{1}{2} d-\frac{1}{2}\right)
$$

The ideal Bose gas has the usual distribution function given by

$$
n_{0}(k)=\left\{\exp \left[\beta\left(k^{2}-\mu\right)\right]-1\right\}^{-1}
$$

where $\mu$ is the chemical potential, and $\beta=\left(k_{\mathrm{B}} T\right)^{-1}$. The critical temperature $T_{c 0}$ is defined to be the temperature below which the ground state starts to become macroscopically occupied, and is evidently just that temperature at which $\mu$ becomes zero. From Eq. (8) one obtains

$$
\begin{aligned}
N & =(2 \pi)^{-d} \int d^{d} k n_{0}(k) \\
& =(2 \pi)^{-d} \int d^{d} k\left[\exp \left(\beta_{c 0} k^{2}\right)-1\right]^{-1} \\
& =\frac{1}{2} K_{d} \Gamma\left(\frac{1}{2} d\right) \zeta\left(\frac{1}{2} d\right) \beta_{c 0}^{-d / 2}
\end{aligned}
$$

and using Eq. (2), the transition temperature in the ideal Bose gas is thus given by

$$
T_{c 0}=\left(4 \pi / k_{\mathrm{B}}\right)\left[N / \zeta\left(\frac{1}{2} d\right)\right]^{2 / d}
$$

From Eqs. (2)-(4) and (9) the parameter $r_{s}$ can thus be expressed as

$$
r_{s}=e^{2} \beta_{c 0}^{1 / 2}\left[\Gamma\left(\frac{1}{2} d+1\right) / \zeta\left(\frac{1}{2} d\right)\right]^{1 / d}
$$

Microscopic calculations on the nonideal many-body system proceed from the one- and two-body thermodynamic Green's functions, and thence 
via the grand partition function $Z_{G}$, defined in terms of the Hamiltonian $\mathscr{H}$ and the total number operator $\mathcal{N}$, as

$$
Z_{G} \equiv e^{\Xi}=\operatorname{Tr}\left[e^{-\beta(x-\mu \cdot r)}\right]
$$

from which one can obtain all of the relevant thermodynamic functions. Alternatively, the most useful second derivatives of the grand partition function can be expressed in terms of fluctuations in the grand canonical ensemble. In the neighborhood of a critical point it is these quantities that become singular, and are hence of special interest. Since the fluctuations are susceptible to direct microscopic derivation, one can avoid the need to explicitly differentiate (a model approximation to) the grand partition function, which can obviously be a delicate procedure close to a critical point. Denoting the grand canonical average of the thermodynamic quantity represented by the operator $\hat{A}$ by

$$
A=\langle\hat{A}\rangle=Z_{\mathrm{G}}^{-1} \operatorname{Tr}\left[e^{-\beta(x-\mu, N)} \hat{A}\right]
$$

and using $\beta$ and $\gamma \equiv \beta \mu$ as the natural independent intensive variables for partial differentiation,

$$
\left(\frac{\partial \Xi}{\partial \beta}\right)_{\gamma} \equiv \Xi_{\mid \beta}=-E ; \quad\left(\frac{\partial \Xi}{\partial \gamma}\right)_{\beta} \equiv \Xi_{\mid \gamma}=N
$$

one can define three independent fluctuations of interest as

$$
\begin{aligned}
\Delta N^{2} & \equiv\left\langle\mathscr{N}^{2}\right\rangle-\langle\mathscr{N}\rangle^{2}=\Xi_{|\gamma| \gamma} \\
\Delta N E & \equiv\langle\mathscr{N} \mathscr{H}\rangle-\langle\mathscr{N}\rangle\langle\mathscr{H}\rangle=-\Xi_{|\beta| \gamma} \\
\Delta E^{2} & \equiv\left\langle\mathscr{H}^{2}\right\rangle-\langle\mathscr{H}\rangle^{2}=\Xi_{|\beta| \beta}
\end{aligned}
$$

The various thermodynamic functions of interest, such as isothermal compressibility $K_{T}$, isobaric expansivity $\alpha_{P}$, and specific heats at constant pressure and volume $C_{P}$ and $C_{V}$, respectively, can all be expressed in terms of the fluctuations defined above. Thus

$$
\begin{aligned}
K_{T} & \equiv-\frac{1}{V}\left(\frac{\partial V}{\partial P}\right)_{T}=\frac{k_{\mathrm{B}} \beta^{2}}{N^{2}} T V \Delta N^{2} \\
\alpha_{P} & \equiv \frac{1}{V}\left(\frac{\partial V}{\partial T}\right)_{P}=\frac{k_{\mathrm{B}} \beta^{2}}{N^{2}}\left(H \Delta N^{2}-N \Delta N E\right) \\
C_{P} & \equiv T\left(\frac{\partial S}{\partial T}\right)_{P}=\frac{k_{\mathrm{B}} \beta^{2}}{N^{2}}\left(H^{2} \Delta N^{2}-2 H N \Delta N E+N^{2} \Delta E^{2}\right) \\
C_{V} & \equiv T\left(\frac{\partial S}{\partial T}\right)_{V}=\frac{k_{\mathrm{B}} \beta^{2}}{N^{2}}\left[N^{2} \Delta E^{2}-N^{2} \frac{(\Delta N E)^{2}}{\Delta N^{2}}\right]
\end{aligned}
$$


where $H$ is the enthalpy, defined as usual by

$$
H=E+P V=T S+\mu N
$$

\section{THE MANY-BODY THEORY FORMALISM}

The thermodynamic Green's functions are defined in terms of the exact "imaginary-time" field operators in the (modified) Heisenberg representation, which are given in terms of the grand canonical Hamiltonian,

$$
\mathscr{K}=\mathscr{H}-\mu \mathscr{N}
$$

as*

$$
\hat{\psi}_{K}(\mathbf{x} \tau)=e^{x_{\tau}} \hat{\psi}(\mathbf{x}) e^{-x_{\tau}}, \quad \hat{\psi}_{K}^{\dagger}(\mathbf{x} \tau)=e^{x_{\tau}} \hat{\psi}^{\dagger}(\mathbf{x}) e^{-x_{\tau}}
$$

The single-particle thermodynamic Green's function is defined as

$$
\begin{aligned}
\mathscr{G}\left(\mathbf{x} \tau ; \mathbf{x}^{\prime} \tau^{\prime}\right) & =-\left\langle T_{\tau}\left[\hat{\psi}_{K}(\mathbf{x} \tau) \hat{\psi}_{K}^{\dagger}\left(\mathbf{x}^{\prime} \tau^{\prime}\right)\right]\right\rangle \\
& \equiv-\operatorname{Tr}\left\{\hat{\rho}_{G} T_{\tau}\left[\hat{\psi}_{K}(\mathbf{x} \tau) \hat{\psi}_{K}^{\dagger}\left(\mathbf{x}^{\prime} \tau^{\prime}\right)\right]\right\}
\end{aligned}
$$

where $\hat{\rho}_{G}$, the statistical operator for the grand canonical ensemble, is given by

$$
\hat{\rho}_{G}=Z_{G}^{-1} \mathrm{e}^{-\beta x}=e^{\beta(\Omega-X)}
$$

and $\Omega$ is the thermodynamic potential,

$$
\Omega=-P V=-\beta^{-1} \Xi
$$

The symbol $T_{\tau}$ orders the operators according to their values of the argument $\tau$, with the smallest at the right.

The two-particle thermodynamic Green's function can be similarly defined, although we shall only need a special case of it, namely the densitydensity correlation function or polarization propagator, defined in terms of the density deviation operator,

$$
\tilde{n}(\mathbf{x})=\hat{n}(\mathbf{x})-\langle\hat{n}(\mathbf{x})\rangle ; \quad \hat{n}(\mathbf{x})=\hat{\psi}^{\dagger}(\mathbf{x}) \hat{\psi}(\mathbf{x})
$$

as

$$
\mathscr{D}\left(\mathbf{x} \tau ; \mathbf{x}^{\prime} \tau^{\prime}\right)=-\left\langle T_{\tau}\left[\tilde{n}_{K}(\mathbf{x} \tau) \tilde{n}_{K}\left(\mathbf{x}^{\prime} \tau^{\prime}\right)\right]\right\rangle
$$

Both $\mathscr{G}$ and $\mathscr{D}$ are periodic in each (real) $\tau$ variable with period $\beta,{ }^{24}$ and since $\mathscr{H}$ is time independent, both functions depend only on the combination $\left(\tau-\tau^{\prime}\right)$, and may hence be expanded in a Fourier series in this variable.

*Note that $\hat{\psi}_{K}^{+}$is defined by Eq. (13), and is not the true adjoint of $\hat{\psi}_{K}$ since $\tau$ is assumed real (hence the term "imaginary time"), although $\hat{\psi}^{\dagger}$ is the adjoint of the field operator $\hat{\psi}$ in the Schrödinger representation. 
We define the Fourier transform by

$$
\begin{aligned}
& \mathscr{P}\left(\mathbf{x} \tau ; \mathbf{x}^{\prime} \tau^{\prime}\right)=(2 \pi)^{-d} \int d^{d} q \beta^{-1} \\
& \times \sum_{n=-\infty}^{\infty}\left\{\exp \left[i \mathbf{q} \cdot\left(\mathbf{x}-\mathbf{x}^{\prime}\right)\right]\right\}\left\{\exp \left[-i v_{n}\left(\tau-\tau^{\prime}\right)\right]\right\} \mathscr{P}\left(\mathbf{q}, v_{n}\right), \\
& v_{n}=2 n \pi \beta^{-1}
\end{aligned}
$$

where $\mathscr{P}$ may stand for either $\mathscr{G}$ or $\mathscr{D}$. The proper self-energy $\Sigma^{*}$ and proper polarization $\mathscr{D}^{*}$ are given by Dyson's equations as

$$
\begin{aligned}
& \mathscr{G}\left(\mathbf{k}, v_{n}\right)=\left[\mathscr{G}_{0}\left(\mathbf{k}, v_{n}\right)^{-1}-\Sigma^{*}\left(\mathbf{k}, v_{n}\right)\right]^{-1} \\
& \mathscr{D}\left(\mathbf{k}, v_{n}\right)=\mathscr{D}^{*}\left(\mathbf{k}, v_{n}\right)\left[1-V(\mathbf{k}) \mathscr{D}^{*}\left(\mathbf{k}, v_{n}\right)\right]^{-1}
\end{aligned}
$$

where $\mathscr{G}_{0}$ is the free thermodynamic single-particle propagator,

$$
\mathscr{G}_{0}\left(\mathbf{k}, v_{n}\right)=\left[i v_{n}-\left(k^{2}-\mu\right)\right]^{-1} e^{i v_{n} \delta}
$$

The quantity $\delta$ is a positive infinitesimal, and the exponential term involving $\delta$ is inserted only when the two ends of the propagator join the same bare interaction line. If necessary the thermodynamic ("imaginary-time") propagators defined above can be related to the corresponding real-time functions by analytic continuation, using the Lehmann representations (Ref. 24, Chapter 9) for the various propagators involved. It is also useful to define a generalized or dressed interaction $\mathscr{U}$ by analogy to Eq. (19) as

$$
\mathscr{U}\left(\mathbf{k}, v_{n}\right)=V(\mathbf{k})\left[1-V(\mathbf{k}) \mathscr{D}^{*}\left(\mathbf{k}, v_{n}\right)\right]^{-1}
$$

The main critical indices of interest can be obtained from the various limiting forms of the thermodynamic propagators. Both $\mathscr{G}\left(\mathbf{k}, v_{n}\right)$ and $\mathscr{D}^{*}\left(\mathbf{k}, v_{n}\right)$ as defined above depend implicitly on the parameters $\beta$ and $\mu$. These parameters in turn are not independent (for fixed density), since particle conservation leads to the expression

$$
N=-\beta^{-1}(2 \pi)^{-d} \int d^{d} k \sum_{n} \mathscr{G}\left(\mathbf{k}, v_{n}\right)
$$

which can be solved to give $\mu$ as a function of $\beta$ (at fixed density). Instead of using either $\beta$ or $\mu$ as independent variable, it is often more convenient to work with a derived parameter $r$ [not to be confused with the small dimensionless parameter $r_{s}$ of Eq. (10)], defined by

$$
r \equiv-\mathscr{G}^{-1}(0,0)=\Sigma^{*}(0,0)-\mu
$$

where $\Sigma^{*}(0,0)$ itself depends implicitly on $\beta$ and $\mu$.

The relevant critical exponents can be obtained from the "static" (i.e., $v_{n}=0$ ) thermodynamic Green's functions, and to make explicit the 
dependence on the parameter $r$, we write

$$
\begin{aligned}
\Sigma^{*}\left(\mathbf{k}, v_{n}=0\right) \equiv & E^{*}(k, r) \\
\mathscr{G}\left(\mathbf{k}, v_{n}=0\right) \equiv & G(k, r)=-\left\{k^{2}+E^{*}(k, r)-E^{*}(0, r)+r\right\}^{-1} \\
& \equiv-\left\{k^{2}+\varepsilon(k, r)\right\}^{-1} \\
\mathscr{D}^{*}\left(\mathbf{k}, v_{n}=0\right) \equiv & D^{*}(k, r)
\end{aligned}
$$

The critical exponents are now defined by the asymptotic form of these correlation functions for small $k$ at zero $r$ (which, as we shall see, corresponds to $T=T_{c}$ ) or for small $r$ at zero $k$. We have

$$
G(k, 0) \sim k^{-2+n} ; \quad D^{*}(k, 0) \sim k^{\bar{\lambda}}
$$

as the leading terms for small $k$ at zero $r$, and

$$
-G(0, r) \equiv r^{-1} \sim\left(\mu_{c}-\mu\right)^{-\gamma} ; \quad D^{*}(0, r) \sim r^{-\bar{\alpha} / \gamma} \sim\left(\mu_{c}-\mu\right)^{-\bar{\alpha}}
$$

as the leading terms for small $r$ (or equivalently, small values of the parameter $\mu_{c}-\mu$ ) at zero $k$, where $\mu_{c}$ is the value $\mu_{c}=\mu\left(\beta_{c}\right)$ of the chemical potential at the critical temperature. These expressions (25) and (26) define the critical exponents $\eta, \gamma, \bar{\lambda}$, and $\bar{\alpha}$. We shall see in Section 7 that the quantity $\mu_{c}-\mu$ is directly proportional to the quantity $T-T_{c}$.

\section{THE SELF-CONSISTENT APPROXIMATION}

We have seen that the critical exponents of interest can be found from a calculation of the propagators $\mathscr{G}$ and $\mathscr{D}^{*}$. Since we are interested in the region around the critical point, where any singularities are expected to manifest themselves, it is important that any calculation be self-consistent. The lowest-order diagrams for $\Sigma^{*}$, written in terms of the free propagator $\mathscr{G}_{0}$ and the bare potential, are the usual Hartree-Fock terms [although in this case there will be no direct or Hartree term, since for our potential $V(q)=0$ ]; and for $\mathscr{D}^{*}$ the lowest-order term is the bubble or ring diagram. As our model for the problem, we take these same diagrams but constructed from the full self-consistent propagator $\mathscr{G}$ and the dressed interaction $\mathscr{U}$, as shown in Fig. 1(c) and 1(d). The four equations represented diagrammatically in Fig. 1 comprise our fully self-consistent approximation to the problem. They represent a coupled set of integral equations for the two quantities of interest, $\mathscr{G}$ and $\mathscr{D}^{*}$. The diagrams of Fig. 1(c) and $1(\mathrm{~d})$ are given respectively by

$$
\begin{gathered}
\Sigma^{*}\left(\mathbf{p}, v_{n}\right)=-\frac{1}{\beta} \sum_{m} \int \frac{d^{d} q}{(2 \pi)^{d}} \mathscr{U}\left(\mathbf{p}+\mathbf{q}, v_{n}+v_{m}\right) \mathscr{G}\left(\mathbf{q}, v_{m}\right) \\
\mathscr{D}^{*}\left(\mathbf{p}, v_{n}\right)=-\frac{1}{\beta} \sum_{m} \int \frac{d^{d} q}{(2 \pi)^{d}} \mathscr{G}\left(\mathbf{p}+\mathbf{q}, v_{n}+v_{m}\right) \mathscr{G}\left(\mathbf{q}, v_{m}\right)
\end{gathered}
$$




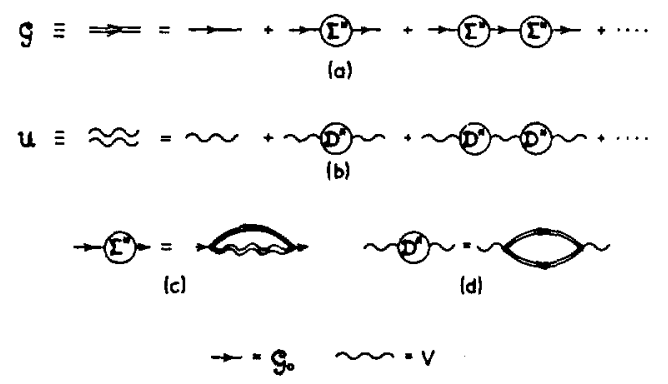

Fig. 1. A diagrammatic representation of the selfconsistent equations.

These two equations, together with Eqs. (18) and (21), now have to be solved self-consistently for $\mathscr{G}$ and $\mathscr{D}^{*}$. As they stand, this is a hopelessly difficult task, but one can make a single approximation at this point that enables the problem to become tractable. This consists in replacing the dressed potential $\mathscr{U}\left(\mathbf{q}, v_{n}\right)$ by its "static" value $\mathscr{U}\left(\mathbf{q}, v_{n}=0\right)$. We shall see that this in turn permits a solution for $\Sigma^{*}\left(\mathbf{p}, v_{n}\right)$ which is independent of $v_{n}$. Making the static approximation in Eq. (27) gives

$$
\Sigma^{*}\left(\mathbf{p}, v_{n}\right)=-\frac{1}{\beta} \int \frac{d^{d} q}{(2 \pi)^{d}} \mathscr{U}(\mathbf{p}+\mathbf{q}, 0) \sum_{m} \frac{e^{i v_{m} \delta}}{i v_{m}-\left[q^{2}+\Sigma^{*}\left(\mathbf{q}, v_{m}\right)-\mu\right]}
$$

which obviously gives a solution independent of $v_{n}$. The frequency sum can now be performed, using the relation ${ }^{24}$.

$$
\lim _{\delta \rightarrow 0^{+}} \sum_{n=-\infty}^{\infty} \frac{e^{i v_{n} \delta}}{i v_{n}-x}=-\frac{\beta}{e^{\beta x}-1}
$$

to give

$$
\begin{aligned}
\Sigma^{*}(p) & =(2 \pi)^{-d} \int d^{d} q \mathscr{U}(\mathbf{p}+\mathbf{q}) n(q) \\
n(q) & =\{\exp [\beta(\varepsilon(q)+r)]-1\}^{-1}
\end{aligned}
$$

where $r$ is defined in Eq. (23), and

$$
\varepsilon(p) \equiv p^{2}+\Sigma^{*}(p)-\Sigma^{*}(0)
$$

Equations (30) and (32) give immediately

$$
\varepsilon(p)=p^{2}+(2 \pi)^{-d} \int d^{d} q \mathscr{U}(\mathbf{q})[n(\mathbf{p}+\mathbf{q})-n(q)]
$$


The frequency sum in Eq. (28) can similarly be performed, to give .

$$
\mathscr{D}^{*}(\mathbf{q}, 0)=\int \frac{d^{d} t}{(2 \pi)^{d}} \frac{n(\mathbf{t}+\mathbf{q})-n(t)}{\varepsilon(\mathbf{t}+\mathbf{q})-\varepsilon(t)}
$$

The total number of particles is found from Eqs. (24) and (29) to be

$$
N=(2 \pi)^{-d} \int d^{d} q n(q)
$$

which verifies that $n(q)$ is just the self-consistent distribution function.

The internal energy of the system can also be found for our model, using the general relation ${ }^{24}$

$$
E=-\beta^{-1}(2 \pi)^{-d} \int d^{d} k \sum_{n} e^{i v_{n} \delta}\left[k^{2}+\frac{1}{2} \Sigma^{*}\left(\mathbf{k}, v_{n}\right)\right] \mathscr{G}\left(\mathbf{k}, v_{n}\right)
$$

With our static approximation for the self-energy, the frequency sum is again immediately calculable, to give

$$
E=(2 \pi)^{-d} \int d^{d} k\left[k^{2}+\frac{1}{2} \Sigma^{*}(k)\right] n(k)
$$

The thermodynamic potential is a little more difficult to obtain since it cannot be found directly from the single-particle propagator, but is given by the general expression ${ }^{25}+$

$$
\begin{aligned}
\mathbf{\Omega}= & \beta^{-1}(2 \pi)^{-d} \int d^{d} p \sum_{l} e^{i v_{l} \delta}\left\{\ln \left[p^{2}+\Sigma^{*}\left(\mathbf{p}, v_{l}\right)-\mu-i v_{l}\right]\right. \\
& \left.+\Sigma^{*}\left(\mathbf{p}, v_{l}\right) \mathcal{G}\left(\mathbf{p}, v_{l}\right)\right\}+\Omega^{\prime} \\
\mathbf{\Omega}^{\prime}= & -\frac{1}{2 \beta} \sum_{n=1}^{\infty} \frac{1}{n}(2 \pi)^{-d} \int d^{d} p \sum_{l} \mathscr{G}\left(\mathbf{p}, v_{l}\right) \Sigma_{s}^{*(n)}\left(\mathbf{p}, v_{l}\right)
\end{aligned}
$$

where $\Sigma_{s}^{*(n)}$ is the total contribution to the self-energy from the $n$ th-order skeleton diagrams in which the bare propagators $\mathscr{G}_{0}$ are replaced by the full propagators. In this context the order of a bare diagram (i.e., one formed only from bare potential lines $V$ and bare propagator lines $\mathscr{G}_{0}$ ) is the number of potential lines appearing in the diagram, and the skeleton diagrams comprise that subset of the bare diagrams which contain no self-energy insertions on any particle line. The self-energy $\Sigma_{s}^{*(n)}$ is thus found by summing all $n$ thorder bare skeleton diagrams in which each of the $2 n-1$ bare propagator lines is first replaced by a full propagator. In our model of keeping only the ring diagrams $\Sigma_{s}^{*(n)}$ has only one contribution in each order, as shown in

tOur Eqs. (38) and (39) differ from Eq. (47) of Ref. 25 by an overall minus sign, which reflects the usual sign difference between the corresponding expressions for boson and fermion systems. 
Fig. 2, given by

$$
\begin{aligned}
\Sigma_{s}^{*(n)}\left(\mathbf{p}, v_{l}\right)= & -\beta^{-1}(2 \pi)^{-d} \int d^{d} q \\
& \sum_{m}[V(\mathbf{q})]^{n}\left[\mathscr{D}^{*}\left(\mathbf{q}, v_{m}\right)\right]^{n-1} \mathscr{G}\left(\mathbf{p}+\mathbf{q}, v_{l}+v_{m}\right)
\end{aligned}
$$

and inserting this expression into Eq. (39) gives

$$
\begin{aligned}
\Omega^{\prime} & =-\frac{1}{2 \beta} \sum_{n=1}^{\infty} \frac{1}{n}(2 \pi)^{-d} \int d^{d} q \sum_{m}\left[V(\mathbf{q}) \mathscr{D}^{*}\left(\mathbf{q}, v_{m}\right)\right]^{n} \\
& =\frac{1}{2 \beta}(2 \pi)^{-d} \int d^{d} q \sum_{m} \ln \left[1-V(\mathbf{q}) \mathscr{D}^{*}\left(\mathbf{q}, v_{m}\right)\right]
\end{aligned}
$$

The frequency sums in Eq. (38) can be performed, since our self-energy is frequency independent. The sum needed for the first term is readily found by replacing $x$ by $\lambda x$ in Eq. (29) and integrating over $\lambda$ in the range $(1, \infty)$, which yields the identity

$$
\lim _{\delta \rightarrow 0^{+}} \sum_{n=-\infty}^{\infty} e^{i v_{n} \delta} \ln \left(x-i v_{n}\right)=\ln \left(1-e^{-\beta x}\right)
$$

Using this relation, we thus find

$$
\begin{aligned}
\Omega & =\beta^{-1}(2 \pi)^{-d} \\
& \times \int d^{d} p\left(\ln \left\{1-\exp \left[-\beta\left(p^{2}+\Sigma^{*}(p)-\mu\right)\right]\right\}-\beta n(p) \Sigma^{*}(p)\right)+\Omega^{\prime}
\end{aligned}
$$

From the thermodynamic potential it is possible to derive any of the other thermodynamic functions, and in particular one can obtain the fluctuations of Eq. (12). It is also possible to check for self-consistency at this stage, by deriving expressions for $N$ and $E$ from $\Omega$ directly, from the thermodynamic relations

$N=-\left(\frac{\partial \Omega}{\partial \mu}\right)_{\beta}, \quad E=\Omega+T S+\mu N=\Omega+\beta\left(\frac{\partial \Omega}{\partial \beta}\right)_{\mu}-\mu\left(\frac{\partial \Omega}{\partial \mu}\right)_{\beta}$

Using Eq. (41) in the first of these expressions gives

$$
N=(2 \pi)^{-d} \int d^{d} p n(p)+\left\{(2 \pi)^{-d} \int d^{d} p\left[\Sigma^{*}(p) \frac{\partial n(p)}{\partial \mu}-\frac{\partial \Omega^{\prime}}{\partial \mu}\right]\right\}
$$

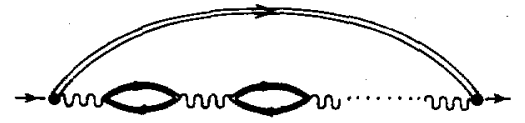

Fig. 2. The only diagram contributing to $\Sigma_{s}^{*(n)}$; containing $n$ interactions and $n-1$ polarization propagators. 
Using Eq. (41), we find

$$
\left(\frac{\partial \Omega^{\prime}}{\partial \mu}\right)_{\beta}=-\frac{1}{2 \beta}(2 \pi)^{-d} \int d^{d} p \sum_{n} \frac{V(\mathbf{p})}{1-V(\mathbf{p}) \mathscr{D}^{*}\left(\mathbf{p}, v_{n}\right)} \frac{\partial \mathscr{D}^{*}\left(\mathbf{p}, v_{n}\right)}{\partial \mu}
$$

To proceed further, we make the same approximation as before, namely that in the denominator of the integrand the polarization propagator is replaced by its static value, to give

$$
\frac{\partial \boldsymbol{\Omega}^{\prime}}{\partial \mu}=-\frac{1}{2 \beta}(2 \pi)^{-d} \int d^{d} p \mathscr{U}(\mathbf{p}) \frac{\partial}{\partial \mu} \sum_{n} \mathscr{D}^{*}\left(\mathbf{p}, v_{n}\right)
$$

From the definition of Eq. (28) we find

$$
\sum_{n} \mathscr{D}^{*}\left(\mathbf{p}, v_{n}\right)=-\beta^{-1}(2 \pi)^{-d} \int d^{d} q \sum_{n} \sum_{m} \mathscr{G}\left(\mathbf{p}+\mathbf{q}, v_{n}+v_{m}\right) \mathscr{G}\left(\mathbf{q}, v_{m}\right)
$$

and since we have made the static approximation for $\mathscr{U}(p)$ above, it is necessary to insert the same frequency convergence factors for the propagators in the above expression as for the bare propagator $\mathscr{G}_{0}$ given in Eq. (20). The two sums can now be performed independently using Eq. (29), to give

$$
\sum_{n} \mathscr{D}^{*}\left(\mathbf{p}, v_{n}\right)=-\beta(2 \pi)^{-d} \int d^{d} q n(\mathbf{q}) n(\mathbf{p}+\mathbf{q})
$$

and hence from Eq. (44),

$$
\frac{\partial \Omega^{\prime}}{\partial \mu}=\frac{1}{2}(2 \pi)^{-2 d} \int d^{d} p \int d^{d} q \mathscr{U}(\mathbf{p})\left[n(\mathbf{q}) \frac{\partial \eta \dot{(p}+\mathbf{q})}{\partial \mu}+n(\mathbf{p}+\mathbf{q}) \frac{\partial n(q)}{\partial \mu}\right]
$$

Substitution from Eq. (30) and a simple shift of integration variables leads to the final result

$$
\frac{\partial \Omega^{\prime}}{\partial \mu}=(2 \pi)^{-d} \int d^{d} p \Sigma^{*}(p) \cdot \frac{\partial n(p)}{\partial \mu}
$$

Inserting this result into Eq. (43) and comparing with Eq. (35) shows that the expression for $N$ derived from $\Omega$ is the same as our previous result.

Similarly, one can show that the expression for $E$ derived from $\Omega$ by Eq. (42) leads to the same expression obtained previously in Eq. (37). It has thus been demonstrated that the expression for $\Omega$ given by Eqs. (38) and (39) and the approximations made to obtain it are consistent with the original set of approximations.

\section{THE QUASIPARTICLE ENERGY SPECTRUM AT $\boldsymbol{T}=\boldsymbol{T}_{\boldsymbol{c}}$}

The quasiparticle distribution function has been found to be given by Eq. (31), and by analogy with the ideal boson gas, the critical temperature $T_{c}$ 
is defined to be that temperature at which the groundstate starts to become macroscopically occupied. The condition for this to happen is evidently that the parameter $r$ should vanish. From Eqs. (21) and (30)-(34) our self-consistent set of equations at the critical point becomes

$$
\begin{aligned}
\varepsilon(k) & =k^{2}+\int \frac{d^{d} q}{(2 \pi)^{d}}[n(\mathbf{k}+\mathbf{q})-n(q)] \frac{V(q)}{1-V(q) D^{*}(q)} \\
D^{*}(q) & =\int \frac{d^{d} k}{(2 \pi)^{d}} \frac{n(\mathbf{k}+\mathbf{q})-n(k)}{\varepsilon(\mathbf{k}+\mathbf{q})-\varepsilon(k)} \\
n(q) & =\left\{\exp \left[\beta_{c} \varepsilon(q)\right]-1\right\}^{-1}
\end{aligned}
$$

The Coulomb potential $V(q)$ is given by Eq. (6). The structure of these equations is still that of an extraordinarily complex set of coupled integral equations for $\varepsilon(q)$. We shall now have to exploit the fact that our system is considered to be at high density in order to introduce a small parameter $v_{c}$ (related to $r_{s}$ ) into the problem. As an ansatz, we try in the above set of equations a solution of the form

$$
\varepsilon(k)=\left(\beta_{c}^{-1 / 2} v_{c}^{\rho}\right)^{2} \varphi\left(k \beta_{c}^{1 / 2} v_{c}^{-\rho}\right)
$$

where the leading order behavior of the function $\varphi(x)$ at small $x$ is assumed to be given by

$$
\lim _{x \rightarrow 0} x^{-\sigma} \varphi(x)=J
$$

which defines the constants $J$ and $\sigma$; and where the dimensionless parameter $v_{c}$ is defined as

$$
\begin{aligned}
v_{c}^{2 \sigma-1} & \equiv Z_{d} e^{2} \beta_{c}^{1 / 2} J^{-2} M \\
& =\frac{Z_{d} M}{J^{2}}\left(\frac{\beta_{c}}{\beta_{c 0}}\right)^{1 / 2}\left[\frac{\zeta\left(\frac{1}{2} d\right)}{\Gamma\left(\frac{1}{2} d+1\right)}\right]^{1 / 2} r_{s}
\end{aligned}
$$

where we have used Eq. (10). The numerical constant $M$ in Eq. (50) is quite arbitrary at this point, and will be chosen for convenience later. The exponent $\rho$ in Eq. (48) is unknown at present, and must be solved for if this form for $\varepsilon(k)$ indeed provides a solution, which must now be checked.

Substitution of Eq. (48) into Eq. (46) gives after a simple change of integration variable

$$
\begin{aligned}
D^{*}\left(v_{c}^{\rho} \beta_{c}^{-1 / 2} s\right)= & \left(v_{c}^{\rho} \beta_{c}^{-1 / 2}\right)^{d-2} \int \frac{d^{d} t}{(2 \pi)^{d}}[\varphi(\mathbf{s}+\mathbf{t})-\varphi(t)]^{-1} \\
& \times\left\{\frac{1}{\exp \left[v_{c}^{2 \rho} \varphi(\mathbf{s}+\mathbf{t})\right]-1}-\frac{1}{\exp \left[v_{c}^{2 \rho} \varphi(t)\right]-1}\right\}
\end{aligned}
$$


We now assume that $\rho>0$, and find the leading order behavior of the above expression in powers of $v_{c}$, by expanding the exponential functions, to give

$$
D^{*}\left(v_{c}^{\rho} \beta_{c}^{-1 / 2} s\right) \underset{v_{c} \rightarrow 0}{\longrightarrow}-v_{c}^{\rho(d-4)} \beta_{c}^{1-d / 2} \int \frac{d^{d} t}{(2 \pi)^{d}} \frac{1}{\varphi(s+t) \varphi(t)}
$$

Since the most important function of $D^{*}(q)$ in Eq. (45) is to screen the Coulomb potential at small momentum, we make the approximation that the above expression is evaluated with the functions $\varphi(x)$ replaced by their limiting forms at small $\dot{x}$ from Eq. (49),

$$
\begin{aligned}
& D^{*}\left(v_{c}^{\rho} \beta_{c}^{-1 / 2} s\right) \underset{v_{c} \rightarrow 0}{\sim}-v_{c}^{\rho(d-4)} \beta_{c}^{1-d / 2} J^{-2} M s^{d-2 \sigma} \\
& M=M(d, \sigma) \equiv(2 \pi)^{-d} \int d^{d} x x^{-\sigma}|1+\mathbf{x}|^{-\sigma}
\end{aligned}
$$

The constant $M$ in Eq. (50) is now chosen to be defined by the integral expression above, which is evaluated in the appendix as Eq. (A12), where it is shown that the integral remains finite only for $\frac{1}{2} d<\sigma<d$ and $d>1$.

The supposed solution for $a(k)$ of Eq. (48) is now substituted into Eq. (45), together with the derived expression for $D^{*}(q)$. After the potential has been replaced by its analytic form from Eq. (6), and after $e^{2}$ has been replaced using Eq. (50), we easily find

$$
\begin{aligned}
\varphi(t)= & t^{2}+\frac{J^{2}}{M} v_{c}^{2 \sigma-1-\rho} \int \frac{d^{d} s}{(2 \pi)^{d}}\left[n\left(v_{c}^{\rho} \beta_{c}^{-1 / 2} \mid s+t\right)-n\left(v_{c}^{\rho} \beta_{c}^{-1 / 2} s\right)\right] \\
& \times \frac{1}{s^{d-1}+v_{c}^{2 \sigma-1-3 \rho s^{d-2 \sigma}}}
\end{aligned}
$$

The exponential functions in the distribution functions are again expanded to leading order in powers of $v_{c}$, to give

$$
\varphi(t)=t^{2}+\frac{J^{2}}{M} v_{c}^{2 \sigma-1-3 \rho} \int \frac{d^{d} s}{(2 \pi)^{d}}\left[\frac{1}{\varphi(s+t)}-\frac{1}{\varphi(s)}\right] \frac{s^{2 \sigma-d}}{s^{2 \sigma-1}+v_{c}^{2 \sigma-1-3 \rho}}
$$

It is apparent at this point that the ansatz of Eq. (48) does indeed solve the original set of coupled integral equations in the high-density limit $\left(v_{c} \rightarrow 0\right)$, if we choose

$$
\rho=\frac{1}{3}(2 \sigma-1)
$$

and if $\varphi(t)$ satisfies the integral equation

$$
\varphi(t)=t^{2}+\frac{J^{2}}{M} \int \frac{d^{d} s}{(2 \pi)^{d}}\left[\frac{1}{\varphi(\mathrm{s}+\mathrm{t})}-\frac{1}{\varphi(s)}\right] \frac{s^{2 \sigma-d}}{s^{2 \sigma-1}+1}
$$


This is still a rather complicated nonlinear integral equation (although admittedly much simpler than the original coupled equations), and it seems unlikely that an exact analytic solution can be found. We shall not inquire whether Eq. (53) actually has a solution, but assuming that a unique solution exists, it still remains to verify the limiting behavior of Eq. (49). After the substitution $\mathbf{s}=t \mathbf{x}$ the limit $t \rightarrow 0$ is taken in Eq. (53), using the limiting form of Eq. (49). It is seen that the limiting form is valid as long as $\sigma<2$, and we obtain an implicit solution for $\sigma$.

$$
M(d, \sigma)=N(d, \sigma) \equiv(2 \pi)^{-d} \int d^{d} x\left[|\mathbf{1}+\mathbf{x}|^{-\sigma}-x^{-\sigma}\right] x^{2 \sigma-d}
$$

It is seen that the constant $J$ has disappeared from this equation, and can only be obtained from the detailed solution to Eq. (53). The restriction $\sigma<2$ is physically reasonable, since for $\sigma \geq 2$ the critical behavior of the charged Bose gas would be the same as for the ideal gas. The integral $N(d, \sigma)$ is evaluated in the appendix as Eq. (A15), where it is shown that it remains finite only under the conditions $d>\max (1, \sigma)$ and $0<\sigma<2$, both of which are already built into our theory. The implicit equation for $\sigma$ of Eq. (54), using Eqs. (A12) and (A15), reduces to

$$
\begin{gathered}
\Gamma\left(\frac{1}{2} d-\frac{1}{2} \sigma\right) \Gamma\left(\sigma-\frac{1}{2} d\right) \Gamma\left(\frac{1}{2} d-\sigma\right) \Gamma\left(\frac{1}{2} d+\frac{1}{2} \sigma\right)=\Gamma\left(\frac{1}{2} \sigma\right) \Gamma(d-\sigma) \Gamma\left(-\frac{1}{2} \sigma\right) \Gamma(\sigma) \\
d>1, \quad \sigma<2, \quad \frac{1}{2} d<\sigma<d
\end{gathered}
$$

The obvious root $\sigma=\frac{1}{3} d$ to the above equation can be discounted immediately, since it falls outside the range of validity. Using the functional relation

$$
\Gamma(x) \Gamma(1-x)=\pi \operatorname{cosec} \pi x
$$

and the recurrence relation of Eq. (A18), it is readily shown that for $d=3$, Eq. (55) reduces to

$$
\cos \pi \sigma=\sigma(1+\sigma) /(7 \sigma-8)(3-\sigma)
$$

It is easily seen that this equation has only one root in the range $(3 / 2,2)$, and numerical solution gives

$$
\sigma \approx 1.890001, \quad \therefore \quad d=3
$$

Investigation of Eq. (55) readily yields that there is no solution for $\sigma$ for $d \leq 2$, and exactly one solution for $2<d<4$, which is most encouraging. It comes as no surprise that there should be no solution for $d \leq 2$ since the ideal gas does not undergo Bose-Einstein condensation in this case either. It is also pleasing that as $d \rightarrow 4$ the solution for $\sigma$ passes smoothly over to the value two for the noninteracting gas. Numerical solution of Eq. (55) gives the 


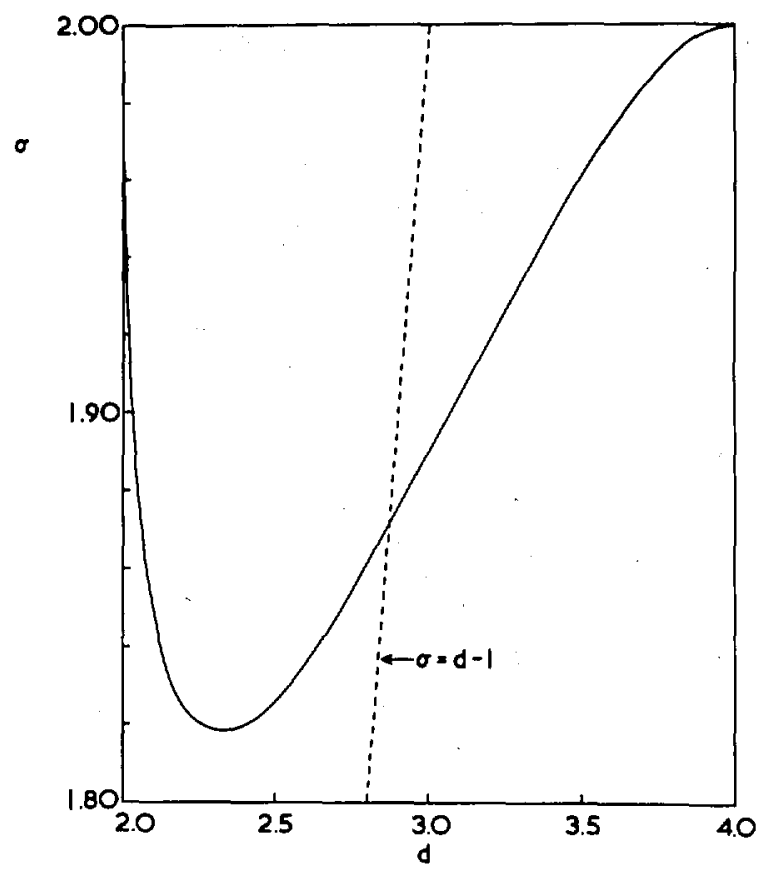

Fig. 3. The exponent $\sigma$ of the low-momentum quasiparticle spectrum, plotted as a function of $d$, from the solution to Eq. (55).

graph of $\sigma$ as a function of dimension shown in Fig. 3. We shall show in Section 7 that a knowledge of $\sigma$ is sufficient to enable us to calculate the various critical indices.

\section{THE TRANSITION TEMPERATURE $\boldsymbol{T}_{c}$}

In the last section the form of the quasiparticle energy spectrum at the critical temperature was found, and its low-momentum behavior was determined explicitly. The critical temperature itself can now be estimated and compared with that of the ideal gas, by use of Eq. (35) and the corresponding equation for the ideal gas,

$$
\begin{aligned}
N= & (2 \pi)^{-d} \int d^{d} k\left\{\exp \left[\beta_{c} \varepsilon(k)\right]-1\right\}^{-1} \\
& =(2 \pi)^{-d} \int d^{d} k\left\{\exp \left(\beta_{c 0} k^{2}\right)-1\right\}^{-1}
\end{aligned}
$$


Subtracting the same quantity from both expressions gives

$$
\begin{aligned}
& \int \frac{d^{d} k}{(2 \pi)^{d}}\left[\frac{1}{\exp \left[\beta_{c} \varepsilon(k)\right]-1}-\frac{1}{\exp \left(\beta_{c} k^{2}\right)-1}\right] \\
= & \int \frac{d^{d} k}{(2 \pi)^{d}}\left[\frac{1}{\exp \left(\beta_{c 0} k^{2}\right)-1}-\frac{1}{\exp \left(\beta_{c} k^{2}\right)-1}\right]
\end{aligned}
$$

Substitution from Eq. (48) for $\varepsilon(k)$ and some simple changes of integration variables readily give

$$
\begin{gathered}
\beta_{c}^{-d / 2} v_{c}^{d \rho} \int \frac{d^{d} t}{(2 \pi)^{d}}\left[\left\{\exp \left[v_{c}^{2 \rho} \varphi(t)\right]-1\right\}^{-1}-\left\{\exp \left(v_{c}^{2 \rho} t^{2}\right)-1\right\}^{-1}\right] \\
=K_{d}\left(\beta_{c 0}^{-d / 2}-\beta_{c}^{-d / 2}\right)^{\frac{1}{2}} \int_{0}^{\infty} d x \frac{x^{d / 2-1}}{e^{x}-1}
\end{gathered}
$$

where we have used Eq. (1). By taking the limit $v_{c} \rightarrow 0$ and evaluating the integral on the right-hand side of the equation, it is easily seen that, to leading order in $v_{c}$, the change in transition temperature is given by

$$
\begin{aligned}
\frac{T_{c}-T_{c 0}}{T_{c 0}} & =\left(\frac{4}{d}\right) \frac{I}{K_{d} \Gamma\left(\frac{1}{2} d\right) \zeta\left(\frac{1}{2} d\right)} v_{c}^{(d-2) \rho} \\
I & \equiv(2 \pi)^{-d} \int d^{d} t\left[t^{-2}-1 / \varphi(t)\right]
\end{aligned}
$$

Using the definition of $v_{c}$ from Eq. (50) and the value of $\rho$ from Eq. (52), we see that, to leading order in $r_{s}$,

$$
\left(T_{c}-T_{c 0}\right) / T_{c 0} \underset{r_{s} \rightarrow 0}{\sim} r_{s}^{(d-2) / 3}
$$

In order to determine the numerical proportionality constant in Eq. (59), it is necessary to evaluate the integral $I$, and it would seem that the function $\varphi(t)$ needs to be known everywhere. The integral $I$ can be rewritten, however, by use of the integral equation (53), which defines $\varphi(t)$, as

$$
I=\frac{J^{2}}{M} \int \frac{d^{d} t}{(2 \pi)^{d}} \frac{1}{t^{2} \varphi(t)} \int \frac{d^{d} s}{(2 \pi)^{d}}\left[\frac{1}{\varphi(s+t)}-\frac{1}{\varphi(s)}\right] \frac{s^{2 \sigma-d}}{s^{2 \sigma-1}+1}
$$

and in this equation it is possibly no worse an approximation to replace the functions $\varphi(s)$ by their limiting forms from Eq. (49) (assuming that the integrals remain finite) than was made in the original derivation of Eq. (53), where a similar approximation was made for $D^{*}(s)$ to arrive at Eq. (51). Making this approximation gives

$$
I=\frac{1}{M} \int \frac{d^{d} t}{(2 \pi)^{d}} t^{-2-\sigma} \int \frac{d^{d} s}{(2 \pi)^{d}}\left[\mathbf{s}+\left.\mathbf{t}\right|^{-\sigma}-s^{-\sigma}\right] \frac{s^{2 \sigma-d}}{s^{2 \sigma-1}+1}
$$


and after interchanging the order of integrations and making a simple change of integration variable $\mathbf{t} \rightarrow \mathrm{x}=s^{-1} \mathbf{t}$ we find

$$
\begin{aligned}
& I=\frac{P}{M} \int \frac{d^{d} s}{(2 \pi)^{d}} \frac{s^{-2}}{s^{2 \sigma-1}+1} \\
& P=P(d, \sigma) \equiv(2 \pi)^{-d} \int d^{d} x x^{-2-\sigma}\left[|1+\mathbf{x}|^{-\sigma}-1\right]
\end{aligned}
$$

The integral $P$ is evaluated in the appendix as Eq. (A17), and the remaining integral in Eq. (61) is evaluated using ${ }^{26}$

$$
\int_{0}^{\infty} d x \frac{x^{\mu-1}}{x^{v}+1}=\frac{\pi}{v} \operatorname{cosec} \frac{\pi \mu}{v} ; \quad v>\mu>0
$$

We finally obtain

$$
I=\frac{\pi K_{d}}{2 \sigma-1}\left(\operatorname{cosec} \frac{\pi(d-2)}{2 \sigma-1}\right) \frac{P}{M}, \quad d>2
$$

in the same range of validity as Eq. (55). Replacing the integrals $P$ and $M$ by their values from the appendix, and substituting Eq. (63) into Eq. (58), gives the result that the leading order change in the transition temperature in powers of $r_{s}$ is, for $2<d<4$,

$$
\begin{aligned}
\frac{T_{c}-T_{c 0}}{T_{c 0}} \approx & -\frac{4 \pi}{\Gamma\left(1+\frac{1}{2} d\right) \zeta\left(\frac{1}{2} d\right)} \frac{(d-1-\sigma)\left(\sigma-\frac{1}{2} d\right)}{\sigma\left(\sigma-\frac{1}{2}\right)(\sigma+2-d)}\left(\operatorname{cosec} \frac{\pi(d-2)}{2 \sigma-1}\right) \\
& \times\left[Z_{d} M\left\{\frac{\zeta\left(\frac{1}{2} d\right)}{\Gamma\left(1+\frac{1}{2} d\right)}\right\}^{1 / d}\right]^{(d-2) / 3}\left(\frac{r_{s}}{J^{2}}\right)^{(d-2) / 3}
\end{aligned}
$$

where we have substituted for $v_{c}$ from Eq. (50). The constants $Z_{d}$ and $M$ are given by Eqs. (7) and (A12), respectively, and only $J$ remains unknown until a more detailed calculation is performed. Since $\sigma$ is determined by Eq. (55), it is easy to see that every factor in Eq. (64) is positive definite in the range $2<d<4$ with the exception of the factor $(d-1-\sigma)$. From Fig. 3, one observes that

$$
d-1-\sigma \gtrless 0, \quad d \gtrless 2.87
$$

This gives $T_{c}<T_{c 0}$ for $4>d>2.87$, and $T_{c}>T_{c 0}$ for $2<d<2.87$, and in particular $T_{c}<T_{c 0}$ for $d=3$. There does not appear to be any obvious physical reason why $T_{c}-T_{c 0}$ should change sign at $d=2.87$, and this is perhaps just an indication that the approximation of replacing $\varphi(x)$ by $J x^{\sigma}$ in Eq. (60) is too drastic, and hence that the numerical factor in Eq. (64) should not be taken too seriously. 
The value of the chemical potential at the critical temperature $\mu_{c}$ can also be evaluated using Eq. (30),

$$
\mu_{c}=\Sigma^{*}(k=0)=\int \frac{d^{d} q}{(2 \pi)^{d}} n(q) \frac{V(q)}{1-V(q) D^{*}(q)}
$$

where the integral is evaluated at $T=T_{c}$. Changing integration variable $q \rightarrow s=v_{c}^{-\rho} \beta_{c}^{1 / 2} q$ and using Eqs. (6), (48), (50), and (51), we obtain after the limit $v_{c} \rightarrow 0$ is taken as before, that to leading order in $v_{c}, \mu_{c}$ is given by

$$
\beta_{c} \mu_{c}=\frac{J^{2}}{M} v_{c}^{2 \rho} \int \frac{d^{d} s}{(2 \pi)^{d}} \frac{1}{\varphi(s)} \frac{s^{2 \sigma-d}}{s^{2 \sigma-1}+1}
$$

Using Eqs. (50) and (52), we see that to leading order in powers of $r_{s}$,

$$
\mu_{c} \underset{r_{s} \rightarrow 0}{\sim} r_{s}^{2 / 3}
$$

independent of $d$ for $2<d<4$. Again the numerical proportionality constant must be evaluated from Eq. (65), which requires an exact knowledge of the function $\varphi(s)$. If we again make the approximation of replacing $\varphi(s)$ by its asymptotic form from Eq. (49), we obtain

$$
\beta_{c} \mu_{c} \approx K_{d} \frac{J}{M} v_{c}^{2 \rho} \int_{0}^{\infty} d s \frac{s^{\sigma-1}}{s^{2 \sigma-1}+1}
$$

and the integral can be performed using Eq. (62), since $\sigma>1$, to give

$$
\beta_{c} \mu_{c} \approx K_{d} \frac{J}{M} v_{c}^{2 \rho} \frac{\pi}{2 \sigma-1} \operatorname{cosec} \frac{\pi \sigma}{2 \sigma-1}
$$

Replacing $v_{c}$ in favor of $r_{s}$ from Eq. (50) gives finally

$\beta_{c} \mu_{c} \approx \frac{\pi K_{d}}{2 \sigma-1}\left(\operatorname{cosec} \frac{\pi \sigma}{2 \sigma-1}\right)\left[Z_{d} M^{-1 / 2}\left\{\frac{\zeta\left(\frac{1}{2} d\right)}{\Gamma\left(1+\frac{1}{2} d\right)}\right\}^{1 / d}\right]^{2 / 3}\left(\frac{r_{s}^{2}}{J}\right)^{1 / 3}$

where the factors $K_{d}, Z_{d}$ and $M$ are known from Eqs. (2), (6), and (A12), respectively, and only $J$ requires a more detailed calculation.

\section{THE CRITICAL INDICES}

From the basic set of coupled equations derived in Section 4 we are now in a position to derive the critical indices defined in Eqs. (25) and (26). Two of them have in fact been essentially derived already. Thus, from Eqs. (24), (32), (48), and (49), we have

$$
G(k, 0) \underset{k \rightarrow 0}{\sim} k^{-\sigma}
$$


which immediately identifies the exponent $\eta$ from Eq. (25) as

$$
\eta=2-\sigma
$$

Similarly from Eqs. (51) and (26), the exponent $\bar{\lambda}$ can also be identified as

$$
\bar{\lambda}=d-2 \sigma
$$

We also observe that $G(k, 0)$ and $D^{*}(k, 0)$ take their asymptotic forms for small $k$ only for $k \ll v_{c}^{\rho} \beta_{c}^{-1 / 2}$, or equivalently from Eqs. (50) and (52), for $k \ll \beta_{c}^{-1 / 2} r_{s}^{1 / 3}$.

It is tempting at this point to assume that our system is just an effectively free $d$-dimensional Bose gas of (infinitely long-lived) quasiparticles with a modified single-particle energy spectrum $\varepsilon(k)=$ const $\times k^{\sigma}$, and to enumerate the remaining critical indices accordingly. Gunton and Buckingham ${ }^{27}$ have evaluated the critical indices for this case, and Panat et al., ${ }^{13}$ in considering the dense, charged Bose gas at $d=3$ only, and using just this prescription, quoted results for their critical indices using this work, substituting the value of $\sigma$ they had found. $\dagger$ This prescription cannot, however, be correct, for two reasons. First, the quasiparticle energy spectrum is manifestly temperature dependent, and is only of the form $\varepsilon(k)=$ const $\times k^{\sigma}$ at $T=T_{c}$ : for $T>T_{c}$ the basic set of equations derived in Section 4 must be investigated anew. Second, even were we to make the approximation that the energy spectrum is independent of temperature (or equivalently, of the parameter $r$ ), the distribution function would still be of the form of Eq. (31), whereas that of the modified ideal gas would have the quantity $-r$ replaced by the chemical potential $\mu$, which would lead to further differences.

Thus, in order to investigate the critical indices $\gamma$ and $\bar{\alpha}$ from Eq. (26), we must perform a similar analysis to that of Section 5 , but at $T>T_{c}$ or equivalently for nonzero values of the parameter $r$.

From Eqs. (31) and (34) we have

where

$$
D^{*}(q, r)=\int \frac{d^{d} k}{(2 \pi)^{d}} \frac{n(\mathbf{k}+\mathbf{q}, r)-n(k, r)}{\varepsilon(\mathbf{k}+\mathbf{q}, r)-\varepsilon(k, r)}
$$

$$
n(k, r)=\{\exp [\beta(\varepsilon(q, r)+r)]-1\}^{-1}
$$

and the dependence on $r$ has been written explicitly. Since we are only interested in the singular part of $D^{*}(q, r)$, the exponential terms in the distribution functions $n(k, r)$ can be expanded as in Section 5 to give

$$
D^{*}(q, r) \approx-\frac{1}{\beta} \int \frac{d^{d} k}{(2 \pi)^{d}} \frac{1}{\varepsilon(k, r)+r} \frac{1}{\varepsilon(\mathbf{k}+\mathbf{q}, r)+r}
$$

†These authors quote a result $\sigma \approx 1.8$ (for $d=3$ ), which may be compared with our result of $\sigma \approx 1.890$ for this case. 
The quasiparticle energy spectrum function $\varepsilon(k, r)$ is given by the solution to

$$
\varepsilon(k, r)=k^{2}+\int \frac{d^{d} q}{(2 \pi)^{d}}[n(\mathbf{k}+\mathbf{q}, r)-n(q, r)] \frac{V(q)}{1-V(q) D^{*}(q, r)}
$$

After the spirit of the assumptions used in deriving scaling relations ${ }^{28.29}$ we make the ansatz that $a(k, r)$ can be written for small values of $r$ in the form

$$
\varepsilon(k, r)+r=r[f(k \xi)]^{-1}, \quad \xi=A r^{-x}
$$

where $\xi$ is a scaling or correlation length, and the function $f(s)$, the constant $A$, and the exponent $x$ are unknown. We have suppressed all of the dependence on the parameter $r_{s}$ (or equivalently $v_{c}$ ) here since it is of no interest for our present purposes. Assuming that $\varepsilon(k, r)$ can be written in the form of Eq. (72), the limiting behavior of the function $f(s)$ can be immediately derived for small and large values of $s$. From the definition of $a(k, r)$ from Eq. (24), we have that $\varepsilon(0, r)=0$, which gives

$$
f(s) \underset{s \rightarrow 0}{\rightarrow} 1
$$

We also have from Eqs. (48) and (49) that for $r$ equal to zero

$$
\varepsilon(k, 0) \equiv \varepsilon(k) \underset{k \rightarrow 0}{\sim} k^{\sigma}
$$

which from Eq. (72) leads to

$$
f(s) \underset{s \rightarrow \infty}{\rightarrow} C s^{-\sigma}
$$

where $C$ is a constant ; and the exponent $x$ must be given by

$$
x=\sigma^{-1}
$$

It remains to prove that the form of $\varepsilon(k, r)$ from Eq. (72) actually solves Eq. (71) for small values of $r$. Inserting Eq. (72) into Eq. (70) gives after a trivial change of integration variable

$$
\begin{aligned}
D^{*}\left(y \xi^{-1}, r\right) & \approx-\beta^{-1} r^{-2} \xi^{-d} G(y), \\
G(y) & =(2 \pi)^{-d} \int d^{d} x f(x) f(\mathbf{x}+\mathbf{y})
\end{aligned}
$$

It is clear that the integral $G(y)$ converges, from Eqs. (73) and (74), and using the fact that $d<2 \sigma$. Expanding the exponential functions as before in the distribution functions $n(q, r)$ in Eq. (71), and inserting the presumed forms of $\varepsilon(k, r)$ from Eq. (72) leads to an equation for $f(x)$ :

$$
\frac{r}{f(k \xi)}-r \approx k^{2}+\frac{1}{\beta} \int \frac{d^{d} q}{(2 \pi)^{d}}[f(|\mathbf{k}+\mathbf{q}| \xi)-f(q \xi)] \frac{1}{V^{-1}(q)-D^{*}(q, r)}
$$


Changing variables to $x=k \xi$ and $y=q \xi$ and inserting the form of $D^{*}(q, r)$ from Eq. (76) gives

$$
\frac{1}{f(x)} \approx 1+\frac{x^{2}}{r \xi^{2}}+\frac{1}{\beta} \int \frac{d^{d} y}{(2 \pi)^{d}}[f(\mathbf{x}+\mathbf{y})-f(y)] \frac{1}{r^{2} \xi^{d} V^{-1}\left(y \xi^{-1}\right)-\beta^{-1} G(y)}
$$

If the limit of the above equation as $r \rightarrow 0$ is now taken, we find, inserting the functional form of $V(q)$ from Eq. (6), and using that $\sigma<2$,

$$
\frac{1}{f(x)}=1-\int \frac{d^{d} y}{(2 \pi)^{d}} \frac{f(\mathbf{x}+\mathbf{y})-f(y)}{G(y)}
$$

Our original ansatz of Eq. (72) has thus been verified, as long as $f(x)$ satisfies the above integral equation. We can now use the proven ansatz to find the remaining critical indices. From Eq. (76) we find immediately

$$
D^{*}(0, r) \underset{r \rightarrow 0}{\sim} r^{-2} \xi^{-d} \sim r^{(d / \sigma-2)}
$$

where the last relation follows from Eqs. (72) and (75). Comparison with Eq. (26) yields the relation between the critical indices $\bar{\alpha}$ and $\gamma$,

$$
\bar{\alpha} / \gamma=(2 \sigma-d) / \sigma
$$

In order to determine the critical index $\gamma$, we use Eqs. (23) and (24), to give

$$
\mu=E^{*}(0, r)-r
$$

Subtracting the critical value $\mu_{c}$ (which occurs at $r=0$ ) gives

$$
\mu: \mu_{c}=E^{*}(0, r)-E^{*}(0,0)-r
$$

and hence from Eq. (26) we find

$$
r+E^{*}(0,0)-E^{*}(0, r) \underset{r \rightarrow 0}{\sim} r^{1 / \gamma}
$$

From Eq. (30) we have

$$
E^{*}(0, r)=\int \frac{d^{d} q}{(2 \pi)^{d}} \frac{n(q, r)}{V^{-1}(q)-D^{*}(q, r)} \approx \frac{1}{\beta} \int \frac{d^{d} q}{(2 \pi)^{d}} \frac{f(q \xi)}{r} \frac{1}{V^{-1}(q)-D^{*}(q, r)}
$$

where the distribution function has been expanded as before, and the relation (72) has been used. The term $V^{-1}(q)$ in the denominator of the integrand can be neglected if we replace the upper limit of the $q$ integration by some finite constant (say, one), since we know that for small $q$ values it is negligible in comparison to $D^{*}(q, r)$, and for high $q$ values the integral is convergent, from our discussion of Section 5 . In order to investigate the leading order behavior 
in powers of $r$, we can thus write

$$
E^{*}(0, r) \approx-\frac{1}{\beta} \int_{0}^{1} \frac{d^{d} q}{(2 \pi)^{d}} \frac{f(q \xi)}{r D^{*}(q, r)}
$$

and hence

$$
E^{*}(0,0)-E^{*}(0, r) \underset{r \rightarrow 0}{\sim} \int_{0}^{1} \frac{d^{d} q}{(2 \pi)^{d}}\left[\frac{f(q \xi)}{r D^{*}(q, r)}-\frac{A^{-\sigma} C q^{-\sigma}}{D^{*}(q, 0)}\right]
$$

where we have used Eqs. (72) and (74). Changing integration variable to $x=q \xi$ and keeping from now on only constants involving $r$ (or $\xi$ ) gives

$$
E^{*}(0,0)-E^{*}(0, r) \sim r^{d / \sigma-1} \int_{0}^{\xi} d^{d} x\left[\frac{f(x)}{D^{*}\left(\xi^{-1} x, r\right)}-\frac{C x^{-\sigma}}{D^{*}\left(\xi^{-1} x, 0\right)}\right]
$$

From Eq. (76) we have

$$
D^{*}\left(\xi^{-1} x, r\right) \sim-r^{d / \sigma-2} G(x)
$$

which by putting $\xi=1$ as a special case and using Eqs. (72) and (75) gives

$$
D^{*}\left(\xi^{-1} x, r\right)=A^{2 \sigma-d} r^{d / \sigma-2} D^{*}\left(x, A^{\sigma}\right)
$$

Inserting this result in Eq. (79) gives

$$
E^{*}(0,0)-E^{*}(0, r) \sim r \int_{0}^{\xi} d^{d} x\left[\frac{f(x)}{D^{*}\left(x, A^{\sigma}\right)}-\frac{C x^{-\sigma}}{D^{*}(x, 0)}\right]
$$

At this point it is tempting to let $r \rightarrow 0(\xi \rightarrow \infty)$, and infer that $E^{*}(0,0)-$ $E^{*}(0, r) \sim r$. This would be incorrect, however, since the remaining integral is not convergent at the upper limit, as we shall now see. From Eq. (80) comparison of the special cases $\xi=1$ and $\xi=x$ gives

$$
D^{*}\left(x, A^{\sigma}\right)=x^{d-2 \sigma} D^{*}\left(1, A^{\sigma} x^{-\sigma}\right)
$$

and hence

$$
E^{*}(0,0)-E^{*}(0, r) \sim r \int_{0}^{\xi} d^{d} x x^{2 \sigma-d}\left[\frac{f(x)}{D^{*}\left(1, A^{\sigma} x^{-\sigma}\right)}-\frac{C x^{-\sigma}}{D^{*}(1,0)}\right]
$$

From Eqs. (74) and( 76) we can write

$$
D^{*}(1, r)-D^{*}(1,0) \underset{r \rightarrow 0}{\sim} r^{-2} \int_{0}^{\xi-1} d^{d} k(k \xi)^{-\sigma}[|\mathbf{k}+1| \xi]^{-\sigma}
$$

where the integral is plainly convergent since $d>\sigma$. We thus find

$$
D^{*}(1, r) \underset{r \rightarrow 0}{\longrightarrow} D^{*}(1,0)+O\left(r^{d / \sigma-1}\right)
$$


The divergence in the integral in Eq. (82), which comes only from the upper limit as $\xi \rightarrow \infty$, is now readily evaluated using this expression and Eq. (74),

$$
\begin{aligned}
E^{*}(0,0)-E^{*}(0, r) & \sim r \int^{\xi} d^{d} x x^{2 \sigma-d} \frac{C x^{-\sigma}}{D^{*}(1,0)}\left(\frac{A}{x}\right)^{d-\sigma} \\
& \sim r \xi^{2 \sigma-d}
\end{aligned}
$$

and hence from Eqs. (72) and (75)

$$
E^{*}(0,0)-E^{*}(0, r) \underset{r \rightarrow 0}{\longrightarrow} O\left(r^{d / \sigma-1}\right)
$$

By comparison with Eq. (78), the critical index $\gamma$ is immediately identified as

$$
\gamma=\sigma /(d-\sigma)
$$

and from Eq. (77)

$$
\bar{\alpha}=(2 \sigma-d) /(d-\sigma)
$$

At this point, we have now identified the four basic critical indices. Two of them, $\gamma$ and $\bar{\alpha}$, are a measure of limiting behavior in the small parameter $\mu_{c}-\mu$ near the critical point, and it is possible to show that in our model this small parameter is directly proportional to $T-T_{c}$. We first define a new function $N^{\prime}(T)$ by

$$
N^{\prime}(T)=(2 \pi)^{-d} \int d^{d} k\left\{\exp \left(\beta A^{\sigma} C^{-1} k^{\sigma}\right)-1\right\}^{-1}
$$

where the constants $A$ and $C$ are given by Eqs. (72) and (74). By a trivial change of integration variable it is obvious that

$$
N^{\prime}(T) / N \approx N^{\prime}(T) / N^{\prime}\left(T_{c}\right)=\left(T / T_{c}\right)^{d / \sigma}
$$

We also have that

$$
\begin{aligned}
N-N^{\prime}(T)= & (2 \pi)^{-d} \int d^{d} k\left[\frac{1}{\exp \{\beta[\varepsilon(k, r)+r]\}-1}\right. \\
& \left.-\frac{1}{\exp \left(\beta A^{\sigma} C^{-1} k^{\sigma}\right)-1}\right] \\
\approx & \beta^{-1}(2 \pi)^{-d} \int d^{d} k\left[\frac{f(k \xi)}{r}-\frac{C}{A^{\sigma} k^{\sigma}}\right]
\end{aligned}
$$

where the second line follows by expanding the exponential functions and 
using Eq. (72). Use of Eq. (74) leads to

$$
\begin{aligned}
N-N^{\prime}(T) & \sim \int_{0}^{\xi^{-1}} d^{d} k\left[\frac{f(k \xi)}{r}-\frac{C}{A^{\sigma} k^{\sigma}}\right] \\
& =A^{-d} r^{d / \sigma-1} \int_{0}^{1} d^{d} x\left[f(x)-C x^{-\sigma}\right]
\end{aligned}
$$

and hence, using Eq. (85),

$$
r \sim\left(T-T_{c}\right)^{\sigma /(d-\sigma)}
$$

Comparison of this result with Eqs. (26) and (83) shows that

$$
\mu_{c}-\mu \sim T-T_{c}
$$

near the critical point.

Before going on to discuss the various scaling relations, it is necessary to digress and discuss the differences between neutral and charged Bose systems. For our charged Bose system the interaction term is given by Eq. (6), where the $k=0$ term is removed by the neutralizing uniform, rigid charge background. The Coulomb interaction is peculiar in that it diverges in the small-momentum limit, which has the effect of drastically altering the behavior of the density-density correlation function $D(k, r)$ in comparison with neutral systems. The proper or irreducible function $D^{*}(k, r)$ defined by Eq. (19) does not behave in the same manner, since this contains no terms with isolated interaction terms which cause the divergence in $D(k, r)$. It is clear from our result of Eq. (69) that for small $k$

$$
D(k, 0) \sim k^{d-1}
$$

Since the $k=0$ term in the interaction potential is removed by the rigid neutralizing background, diagrams contributing to thermodynamic quantities cannot contain isolated interaction lines. As a result, the isothermal compressibility is determined by $D^{*}(0, r)$ and not from $D(0, r)$ as for neutral systems. Thus the relevant critical indices $(\bar{\lambda}$ and $\bar{\alpha})$ for the charged gas are obtained from $D^{*}(k, r)$ as we have done, and in particular the divergence in the isothermal compressibility or specific heat at constant pressure $C_{P}$ near the critical temperature $T_{c}$ is specified by the exponent $-\bar{\alpha}$. For the "equivalent" neutral gas with the same shielded Coulomb interaction of Eq. (21) the only difference is that the isolated interaction lines are not removed, and one can define new functions $G_{n}(k, r)$ and $D_{n}(k, r)$ for this case. The only difference between $G_{n}$ and $G$ is that $G_{n}$ will contain an extra "Hartree" term in its self-energy. It is obvious that this term can be absorbed into the chemical potential $\mu$, and will not affect the relevant critical exponents (although it will cause the transition temperature $T_{c n}$ for the equivalent neutral 
gas to differ from $T_{c}$ ). Thus the exponents $\gamma$ and $\eta$ will be unchanged. The relation between the density-density correlation functions is not so trivial. $\mathrm{Ma}^{12,29}$ has shown, however, that there is a relation between $D_{n}$ and $D^{*}$ of the form

$$
D_{n}(k, r)=\frac{e^{2} D^{*}(k, r)-s^{\prime \prime}}{\left(e^{\prime \prime 2} / s^{\prime \prime}\right) e^{2} D^{*}(k, r)}
$$

where $e^{\prime \prime}$ and $s^{\prime \prime}$ are constants which remain finite both at zero momentum and at $T=T_{c}$. It is clear that we thus have the result

$$
D_{n}(k, r) \sim \text { const }-\left[\text { const } / D^{*}(k, r)\right]
$$

as either $k \rightarrow 0$ or $r \rightarrow 0$. Thus, defining the critical indices for the neutral gas $\alpha$ and $\lambda$ from $D_{n}(k, r)$ by relations analogous to Eqs. (25) and (26), we have

$$
\begin{aligned}
& \alpha=-\bar{\alpha}=-(2 \sigma-d) /(d-\sigma)<0 \\
& \lambda=-\bar{\lambda}=2 \sigma-d>0
\end{aligned}
$$

The various general scaling relations of Widom ${ }^{30}$ and Kadanof ${ }^{31}$ should be tested on the functions $G_{n}$ and $D_{n}$ (or equivalently on the set of critical indices $\gamma, \eta, \alpha$, and $\lambda$ ), rather than on the functions $G$ and $D^{*}$ which are peculiar to the Coulomb problem, for the reasons already cited. Two of the scaling relations cited by these authors, which can be derived ${ }^{28}$ by assuming scaling for both functions $G_{n}$ and $D_{n}$, are, respectively,

$$
\gamma=v(2-\eta) \text { and }-\alpha=v \lambda
$$

where $v$ is a new critical exponent (not to be confused with our previous small parameter $v_{c}$ ) that describes the behavior of the correlation length $\xi$ near the critical point,

$$
\xi \sim\left(\mu_{c}-\mu\right)^{-v}
$$

From our previous discussion of the exact solution, and comparing this definition with Eqs. (26), (72), and (75), $v$ can be identified as

$$
v=\gamma \sigma^{-1}=(d-\sigma)^{-1}
$$

Using this result, and our previously derived values of the critical exponents it is clear that the two simple scaling relations of Eq. (88) are satisfied identically by our model. It is also trivially verifiable that our model satisfies the "hyperscaling" relation of Widom ${ }^{30}$ and Kadanoff, ${ }^{31}$

$$
2-\alpha=v d
$$

In this context we note that whereas $\bar{\alpha}$ and $\lambda$ also satisfy the simple scaling relations (88), they will not satisfy the "hyperscaling" relation (89), as is 
expected from our discussion above. These same authors also derive a scaling relation

$$
\alpha^{\prime}+2 \beta+\gamma^{\prime}=2
$$

where $\alpha^{\prime}$ and $\gamma^{\prime}$ are defined in the region $T<T_{c}$ exactly as $\alpha$ and $\gamma$ are defined for $T>T_{c}$; and $2 \beta$ is the critical exponent defining how $N_{0}$, the number of condensed particles, approaches zero as $T \rightarrow T_{c}$,

$$
N_{0} \sim\left(T_{c}-T\right)^{2 \beta}
$$

If we assume the relation (91) to be true, and also assume that $\alpha^{\prime}=\alpha$, and $\gamma^{\prime}=\gamma$, we find

$$
\beta=\frac{1}{2}
$$

which comes as no surprise, and is compatible with Eq. (85) extended to temperatures $T<T_{c}$ when we make the identification $N_{0}(T)=N-N^{\prime}(T)$. Under the same assumptions of scaling and continuity of the critical exponents across the transition, Josephson ${ }^{32}$ has shown that the exponent $\zeta$, which describes how the superfluid density $\rho_{s}$ approaches zero as $T \rightarrow T_{c}$, $\rho_{s} \sim\left(T_{c}-T\right)^{\zeta}$, can be given by

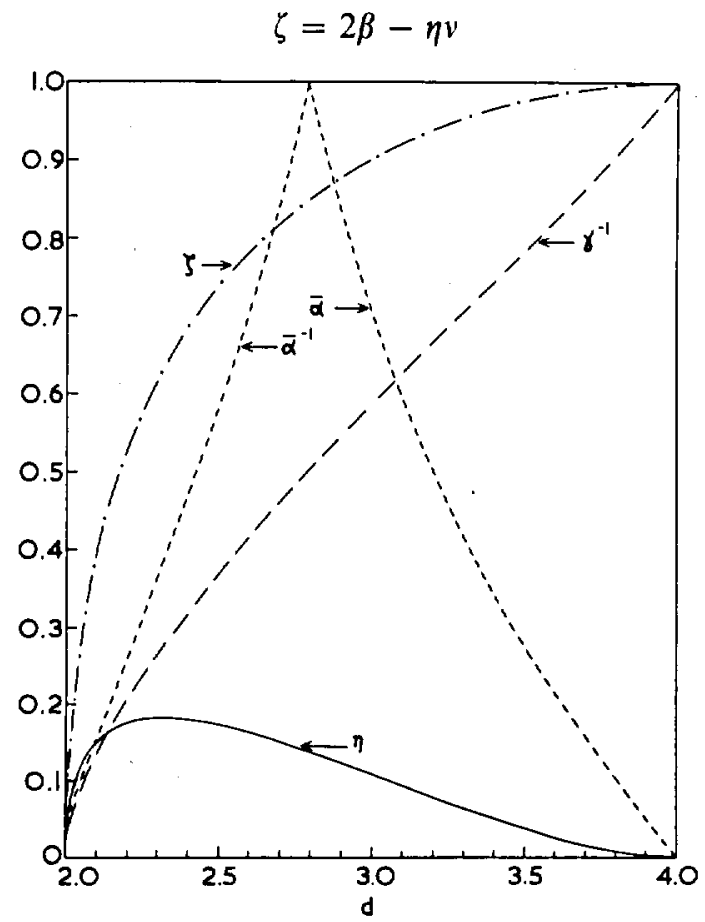

Fig. 4. The critical indices for the high-density charged Bose gas. 
From the values of the exponents derived above we find that for our model

$$
\zeta=(d-2) /(d-\sigma)
$$

Using the solution to Eq. (55), various critical exponents found above are plotted in Fig. 4 as a function of the dimensionality $d$ of the system.

\section{SUMMARY}

The behavior of a charged $d$-dimensional Bose gas at high density and in the presence of a uniform background of opposite charge to ensure electrical neutrality has been examined in the temperature range close to and above the critical temperature for the onset of Bose-Einstein condensation. It has been demonstrated that in this density range the most important critical behavior can be explained in terms of a fully self-consistent version of Hartree-Fock theory based on the interaction screened by the self-consistent static polarization insertions formed from the ring diagrams (random-phase approximation). The approximation was shown to be consistent with the various exact relationships between the thermodynamic functions of the system and the Green's functions calculated assuming the approximations. The quasiparticle spectrum was examined at the critical temperature $T_{c}$, and the functional dependence on the density of the system was calculated. The exact form of the spectrum as a function of momentum was related to the solution of a derived integral equation, and the limiting form at low momenta was shown to be $\varepsilon(k) \rightarrow A k^{\sigma}$, and the exponent $\sigma$ was solved for explicitly as a function of dimension.

The critical temperature itself and the chemical potential at this temperature were related to the quasiparticle spectrum, and the leading order behavior (in powers of the density) of the quantities was found explicitly. The numerical multiplicative constants were shown to depend on a knowledge of the spectrum for all values of the momentum, but approximate expressions were derived from the low-momentum asymptotic form.

Finally, the self-consistent treatment was extended to temperatures above $T_{c}$, and the critical exponents were derived and shown to depend on a knowledge of $\sigma$ only. Table I summarizes the results obtained for the critical indices. The system was shown explicitly to obey exact scaling, and the usual scaling relations were verified.

In conclusion it should be pointed out that the whole treatment has been confined to the region $T \geq T_{c}$, and to leading order in the parameter $r_{s}$ (or equivalently, the inverse of the density). The extension to temperatures below the critical point is not difficult, since the usual well-known many-body theory techniques can be employed to take care of the macroscopic occupation of the condensate. The extension to higher-order terms in the parameter 
TABLE I

The Critical Indices for the Charged Bose Gas in $d$ Dimensions

\begin{tabular}{|c|c|c|c|c|}
\hline Quantity & $\begin{array}{c}\text { Behavior as } \\
k \rightarrow 0 \text { or } T \rightarrow T_{c}\end{array}$ & $\begin{array}{c}\text { Critical } \\
\text { index }\end{array}$ & $\begin{array}{c}\text { Value in terms } \\
\text { of } d \text { and } \sigma\end{array}$ & $\begin{array}{l}\text { Value } \\
(d=3)\end{array}$ \\
\hline $\begin{array}{l}G(k, 0) \\
G(0, r) \\
D^{*}(k, 0) \\
D^{*}(0, r) \\
\xi \\
N_{0}^{1 / 2} \\
\rho_{s}\end{array}$ & $\begin{array}{c}k^{-2+\eta} \\
\left(T-T_{c}\right)^{-\gamma} \\
k^{x^{\lambda}} \\
\left(T-T_{c}\right)^{-\tilde{a}} \\
\left(T-T_{c}\right)^{-v} \\
\left(T_{c}-T\right)^{\beta} \\
\left(T_{c}-T\right)^{\zeta} \\
T_{c 0} \underset{r_{s}^{x}}{\sim r_{s}^{y}}\end{array}$ & $\begin{array}{c}\eta \\
\gamma=-\lambda \\
\lambda=-\alpha \\
\bar{\alpha}=-\alpha \\
y \\
\beta \\
\zeta \\
x \\
y\end{array}$ & $\begin{array}{c}2-\sigma \\
\sigma /(d-\sigma) \\
d-2 \sigma \\
(2 \sigma-d) /(d-\sigma) \\
1 /(d-\sigma) \\
1 / 2 \\
(d-2) /(d-\sigma) \\
(d-2) / 3 \\
2 / 3\end{array}$ & $\begin{array}{c}0.1100 \\
1.7027 \\
-0.7800 \\
0.7027 \\
0.9009 \\
0.5 \\
0.9009 \\
1 / 3 \\
2 / 3\end{array}$ \\
\hline
\end{tabular}

$r_{s}$ presents a more difficult problem, since more terms in the expansion for the proper self-energy and proper polarization must be included. It is relatively trivial to isolate those terms that will contribute to next highest order in (fractional) powers of $r_{s}$, but the requirement of self-consistency now becomes more complicated. This author is presently interested in such an extension of these results.

\section{APPENDIX}

In this appendix we shall evaluate the various $d$-dimensional integrals occurring in the text.

\section{Fourier Transform of $V(r)$}

The interaction potential is taken to be

$$
V(r)=e^{2} r^{-1}
$$

and in order to define its Fourier transform, we employ the standard artifice of introducing a spatial cutoff:

$$
\begin{aligned}
V(k) & =\lim _{\mu \rightarrow 0} V(k ; \mu) \\
V(k ; \mu) & =\int d^{d} r \exp (i \mathbf{k} \cdot \mathbf{r}-\mu r) e^{2} r^{-1} \\
& =e^{2} K_{d-1}(2 \pi)^{d-1} \int_{0}^{\infty} r^{d-2} e^{-\mu r} d r \int_{0}^{\pi} \sin ^{d-2} \theta \cos (k r \cos \theta) d \theta \\
& =e^{2} K_{d-1}(2 \pi)^{d-1} \int_{0}^{\infty} r^{d-2} e^{-\mu r} d r \int_{0}^{1} 2\left(1-u^{2}\right)^{d / 2-3 / 2} \cos (k r u) d u
\end{aligned}
$$


where $K_{d-1}$ is given by Eq. (2). The integration over $u \equiv \cos \theta$ in Eq. (A2) can now be performed ${ }^{33}$ to give

$$
\int_{0}^{1} 2\left(1-u^{2}\right)^{d / 2-3 / 2} \cos (k r u) d u=\pi^{1 / 2} 2^{d / 2-1} \Gamma\left(\frac{1}{2} d-\frac{1}{2}\right)(k r)^{1-d / 2} J_{d / 2-1}(k r)
$$

and hence

$V(k ; \mu)=e^{2} k^{1-d} K_{d-1} \pi^{1 / 2}(2 \pi)^{d-1} 2^{d / 2-1} \Gamma\left(\frac{1}{2} d-\frac{1}{2}\right) \int_{0}^{\infty} d x x^{d / 2-1} J_{d / 2-1}(x) e^{-\mu x / k}$

The last integration can now be performed (Ref. 33, 6.8.6, p. 327) to yield

$$
\begin{aligned}
V(k ; \mu)= & e^{2} k^{1-d} K_{d-1} \pi^{1 / 2}(2 \pi)^{d-1} \frac{\Gamma(d-1)}{\Gamma\left(\frac{1}{2} d\right)}\left(\frac{\mu}{k}\right)^{1-d} \\
& \times{ }_{2} F_{1}\left(\frac{1}{2} d-\frac{1}{2}, \frac{1}{2} d ; \frac{1}{2} d ;-k^{2} / \mu^{2}\right)
\end{aligned}
$$

and the hypergeometric function in Eq. (A3) can be reduced by using the relation $^{34}$

$$
{ }_{2} F_{1}(a, b ; b ; z)=(1-z)^{-a}
$$

The final expression is simplified by using the duplication formula (Ref. 34, 6.1 .18$, p. 256) for the gamma function

$$
\Gamma(2 z)=\pi^{-1 / 2} 2^{2 z-1} \Gamma(z) \Gamma\left(z+\frac{1}{2}\right)
$$

and by substituting from Eq. (2) for $K_{d-1}$ we finally get

$$
V(k)=\lim _{\mu \rightarrow 0} e^{2} \Gamma\left(\frac{1}{2} d-\frac{1}{2}\right)\left(\frac{4 \pi}{k^{2}+\mu^{2}}\right)^{d / 2-1 / 2}
$$

\section{The Integral $M(d, \sigma)$}

The integral $M(d, \sigma)$ is defined in Eq. (51) as

$$
M \equiv M(d, \sigma)=(2 \pi)^{-d} \int d^{d} x x^{-\sigma}|\mathbf{1}+\mathbf{x}|^{-\sigma}
$$

and using Eqs. (1) and (2), hence

$$
M=(2 \pi)^{-1} K_{d-1} \int_{0}^{\pi} d \theta \sin ^{d-2} \theta \int_{0}^{\infty} d x x^{d-\sigma-1}\left(1+2 x \cos \theta+x^{2}\right)^{-\sigma / 2}
$$

The integration over the radial coordinate $x$ can be performed with the aid 
of the relation (Ref. 33, 6.2.22, p. 310)*

$$
\begin{aligned}
& \int_{0}^{\infty} d x x^{s-1}\left(1+2 x \cos \theta+x^{2}\right)^{-v} \\
&=2^{v-1 / 2} \Gamma\left(v+\frac{1}{2}\right) B(s, 2 v-s)(\sin \theta)^{1 / 2-v} P_{s-v-1 / 2}^{1 / 2-v}(\cos \theta), \\
& 0<s<2 v
\end{aligned}
$$

where the beta function is given by

$$
B(x, y)=\Gamma(x) \Gamma(y) / \Gamma(x+y)
$$

Use of Eq. (A8) in Eq. (A7) gives

$$
\begin{aligned}
M= & (2 \pi)^{-1} K_{d-1} 2^{\sigma / 2-1 / 2} \Gamma\left(\frac{1}{2} \sigma+\frac{1}{2}\right) B(d-\sigma, 2 \sigma-d) \\
& \times \int_{0}^{\pi} d \theta(\sin \theta)^{d-\sigma / 2-3 / 2} P_{d-3 \sigma / 2-1 / 2}^{1 / 2-\sigma / 2}(\cos \theta), \quad 2 \sigma>d>\sigma
\end{aligned}
$$

The angular integration can now be performed (Ref. $34,8.14 .16$, p. 338), to give

$$
\begin{array}{r}
\int_{0}^{\pi} d \theta(\sin \theta)^{d-\sigma / 2-3 / 2} P_{d-3 \sigma / 2-1 / 2}^{1 / 2-\sigma / 2}(\cos \theta) \\
=2^{1 / 2-\sigma / 2} \pi \frac{\Gamma\left(\frac{1}{2} d-\frac{1}{2}\right) \Gamma\left(\frac{1}{2} d-\frac{1}{2} \sigma\right)}{\Gamma(d-\sigma) \Gamma\left(\frac{1}{2} \sigma\right) \Gamma\left(\frac{1}{2} d-\frac{1}{2} \sigma+\frac{1}{2}\right) \Gamma\left(\sigma-\frac{1}{2} d+\frac{1}{2}\right)}, \\
d>\max (1, \sigma)
\end{array}
$$

and repeated use of the duplication formula (A5) gives the final expression

$$
M(d, \sigma)=(4 \pi)^{-d / 2} \frac{\Gamma^{2}\left(\frac{1}{2} d-\frac{1}{2} \sigma\right) \Gamma\left(\sigma-\frac{1}{2} d\right)}{\Gamma^{2}\left(\frac{1}{2} \sigma\right) \Gamma(d-\sigma)}, \quad \frac{1}{2} d<\sigma<d ; d>1
$$

\section{The Integral $N(d, \sigma)$}

The integral $N(d, \sigma)$ is defined in Eq. (54) as

$$
N \equiv N(d, \sigma)=(2 \pi)^{-d} \int d^{d} x\left[|\mathbf{1}+\mathbf{x}|^{-\sigma}-x^{-\sigma}\right] x^{2 \sigma-d}
$$

\footnotetext{
*Note that the power of $\sin \theta$ is erroneously given as the negative of the correct result. This error
} is rectified in our Eq. (A8). 
After the simple change of integration variable, $x \rightarrow y=-1-x$ in the first term this becomes

$$
\begin{aligned}
N= & (2 \pi)^{-d} \int d^{d} y\left[|\mathbf{1}+\mathbf{y}|^{2 \sigma-d}-y^{2 \sigma-d}\right] y^{-\sigma} \\
= & (2 \pi)^{-1} K_{d-1} \int_{0}^{\pi} d \theta \sin ^{d-2} \theta \int_{0}^{\infty} d y \\
& \times y^{d-\sigma-1}\left[\left(1+2 y \cos \theta+y^{2}\right)^{\sigma-d / 2}-y^{2 \sigma-d}\right] \\
= & (2 \pi)^{-1} K_{d-1} \int_{0}^{\pi} d \theta \sin ^{d-2} \theta \int_{0}^{\infty} d x \\
& \times x^{-1-\sigma}\left[\left(1+2 x \cos \theta+x^{2}\right)^{\sigma-d / 2}-1\right]
\end{aligned}
$$

where the substitution $y=x^{-1}$ has been made. The radial integration can now be performed as above for the evaluation of $M(d, \sigma)$, using a simple extension of the relation given from Ref. 33 as Eq. (A8). This integral is most readily evaluated by converting it into a contour integral around the branch cuts. The second term in the square brackets of Eq. (A13) then merely ensures that the contribution from the infinitesimal circle about the branch point at the origin vanishes. Hence, we find

$$
\begin{aligned}
\int_{0}^{\infty} d x & x^{-1-\sigma}\left[\left(1+2 x \cos \theta+x^{2}\right)^{\sigma-d / 2}-1\right] \\
= & 2^{d / 2-\sigma-1 / 2} \Gamma\left(\frac{1}{2} d-\sigma+\frac{1}{2}\right) B(-\sigma, d-\sigma)(\sin \theta)^{1 / 2-d / 2+\sigma} \\
& \times P_{-d / 2-1 / 2}^{1 / 2-d / 2+\sigma}(\cos \theta), \quad 0<\sigma<d ; \quad \sigma<2
\end{aligned}
$$

and substitution into Eq. (A13) yields

$$
\begin{aligned}
N= & (2 \pi)^{-1} K_{d-1} 2^{d / 2-\sigma-1 / 2} \Gamma\left(\frac{1}{2} d-\sigma+\frac{1}{2}\right) B(-\sigma, d-\sigma) \\
& \times \int_{0}^{\pi} d \theta(\sin \theta)^{d / 2+\sigma-3 / 2} P_{d / 2-1 / 2}^{1 / 2-d / 2+\sigma}(\cos \theta)
\end{aligned}
$$

The angular integration can now be performed as above, to give

$$
\begin{aligned}
N= & \frac{1}{2} K_{d-1} \Gamma\left(\frac{1}{2} d-\sigma+\frac{1}{2}\right) B(-\sigma, d-\sigma) \\
& \times \frac{\Gamma\left(\frac{1}{2} d-\frac{1}{2}\right) \Gamma(\sigma)}{\Gamma\left(\frac{1}{2} d+\frac{1}{2} \sigma\right) \Gamma\left(\frac{1}{2} \sigma\right) \Gamma\left(\frac{1}{2} d-\frac{1}{2} \sigma+\frac{1}{2}\right) \Gamma\left(\frac{1}{2}-\frac{1}{2} \sigma\right)}, \quad d>1 ; \sigma>0
\end{aligned}
$$

This expression is simplified by repeated use of the duplication formula (A5) to give the final result,

$$
\begin{aligned}
& N(d, \sigma)=(4 \pi)^{-d / 2} \frac{\Gamma\left(-\frac{1}{2} \sigma\right) \Gamma(\sigma) \Gamma\left(\frac{1}{2} d-\frac{1}{2} \sigma\right)}{\Gamma\left(\frac{1}{2} d-\sigma\right) \Gamma\left(\frac{1}{2} d+\frac{1}{2} \sigma\right) \Gamma\left(\frac{1}{2} \sigma\right)}, \\
& d>\max (1, \sigma) ; \quad 0<\sigma<2
\end{aligned}
$$




\section{The Integral $P(d, \sigma)$}

The integral $P(d, \sigma)$ is defined in Eq. (61) as

$$
\begin{aligned}
P \equiv P(d, \sigma)= & (2 \pi)^{-d} \int d^{d} x x^{-2-\sigma}\left[|\mathbf{1}+\mathbf{x}|^{-\sigma}-1\right] \\
= & (2 \pi)^{-1} K_{d-1} \int_{0}^{\pi} d \theta \sin ^{d-2} \theta \\
& \times \int_{0}^{\infty} d x x^{d-\sigma-3}\left[\left(1+2 x \cos \theta+x^{2}\right)^{-\sigma / 2}-1\right]
\end{aligned}
$$

which can be evaluated by exactly the same means as used for $N(d, \sigma)$ above. We quote only the final result,

$$
\begin{aligned}
& P(d, \sigma)=(4 \pi)^{-d / 2} \frac{2(2 \sigma-d)(d-\sigma-1)}{\sigma(d-\sigma-2)} \frac{\Gamma^{2}\left(\frac{1}{2} d-\frac{1}{2} \sigma\right) \Gamma\left(\sigma-\frac{1}{2} d\right)}{\Gamma^{2}\left(\frac{1}{2} \sigma\right) \Gamma(d-\sigma)} \\
& d>\max (1, \sigma), \quad \sigma>\max (0, d-2)
\end{aligned}
$$

where we have used the functional relation

$$
\Gamma(z+1)=z \Gamma(z)
$$

\section{REFERENCES}

1. L. L. Foldy, Phys. Rev. 124, 649 (1961); 125, 2208 (1962).

2. M: Gell-Mann and K. A. Brueckner, Phys. Rev. 106, 364 (1957).

3. N. N. Bogolyubov, J. Phys. (USSR) 11, 23 (1947).

4. M. Girardeau, Phys. Rev. 127, 1809 (1962).

5. B. W. Ninham, Nucl. Phys. 53, 685 (1964).

6. D. K. Lee and E. Feenberg, Phys. Rev. A 137, 731 (1965).

7. K. A. Brueckner, Phys. Rev. 156, 204 (1967).

8. S.-K. Ma and C.-W. Woo, Phys. Rev. 159, 165 (1967); C.-W. Woo and S.-K. Ma, Phys. Rev. $159,176(1967)$.

9. M. Schick and T. M. Wu, Phys. Rev. 177, 313 (1969).

10. E. W. Montroll and J. C. Ward, Phys. Fluids 1, 55 (1958).

11. A. L. Fetter, Ann. Phys. (N.Y.) 64, 1 (1971).

12. S.-K. Ma, Phys. Rev. Letters 29, 1311 (1972).

13. P. V. Panat, H. E. DeWitt, and J. C. Garrison, Phys. Letters 43A, 130 (1973).

14. V. L. Ginzberg, Usp. Fiz. Nauk 48, 25 (1952) [German transl.: Fortschr. Phys. 1, 101 (1953)].

15. M. R. Schafroth, Phys. Rev. 100, 463 (1955).

16. M. J. Stephen, Proc. Phys. Soc. (London) 79, 994 (1962).

17. A. L. Fetter, Ann. Phys. (N.Y.) 60, 464 (1970).

18. J. Bardeen, L. N. Cooper, and J. R. Schrieffer, Phys, Rev. 108, 1175 (1957).

19. B. W. Ninham, Phys. Letters 4, 278 (1963).

20. V. L. Ginzburg, Comments Astrophys. Space Phys. 1, 81 (1969).

21. L. P. Kadanoff et al., Rev. Mod. Phys. 39, 395 (1967).

22. M. E. Fisher, Rep. Progr. Phys. 30, 615 (1967). 
23. K. G. Wilson, Phys. Rev. Letters 28, 548 (1972).

24. A. L. Fetter and J. D. Walecka, Quantum Theory of Many-Particle Systems (McGraw-Hill, New York, 1971), Chapter 7.

25. J. M. Luttinger and J. C. Ward, Phys. Rev. 118, 1417 (1960).

26. H. B. Dwight, Tables of Integrals and Other Mathematical Data, 4th ed. (Macmillan, New York, 1961), p. 213, 856.08.

27. J. D. Gunton and M. J. Buckingham, Phys. Rev. 166, 152 (1968).

28. B. I. Halperin and P. C. Hohenberg, Phys. Rev. 177, 952 (1969).

29. S.-K. Ma, Phys. Rev. A7, 2172 (1973).

30. B. Widom, J. Chem. Phys. 43, 3898, 3892 (1965).

31. L. P. Kadanoff, Physics 2, 263 (1966).

32. B. D. Josephson, Phys. Letters 21, 608 (1966).

33. A. Erdelyi et al., Table of Integral Transforms (McGraw-Hill, New York, 1954), Vol. 1, 1.3.8, p. 11 .

34. M. Abramowitz and I. A. Stegun (eds.), Handbook of Mathematical Functions, Appl. Math. Ser. 55, (U.S. Govt. Printing Office, Washington, D.C., 1964), 15.1.8, p. 556. 